\title{
A characterization of higher rank symmetric spaces via bounded cohomology
}

\author{
Mladen Bestvina and Koji Fujiwara *
}

March 23, 2010

\begin{abstract}
Let $M$ be complete nonpositively curved Riemannian manifold of finite volume whose fundamental group $\Gamma$ does not contain a finite index subgroup which is a product of infinite groups. We show that the universal cover $\tilde{M}$ is a higher rank symmetric space iff $H_{b}^{2}(M ; \mathbb{R}) \rightarrow$ $H^{2}(M ; \mathbb{R})$ is injective (and otherwise the kernel is infinite-dimensional). This is the converse of a theorem of Burger-Monod. The proof uses the celebrated Rank Rigidity Theorem, as well as a new construction of quasi-homomorphisms on groups that act on $C A T(0)$ spaces and contain rank 1 elements.
\end{abstract}

\section{Contents}

1 Introduction $\quad[2$

2 Axioms 5

3 B-contracting segments 6

4 Quasi-homomorphisms 11

4.1 Expressways . . . . . . . . . . . . . . . 12

4.2 Construction of quasi-homomorphisms . . . . . . . . . . 14

5 Rank 1 isometries $\quad 16$

5.1 Characterization when $X$ is a proper $C A T(0)$ space . . . . 17

5.2 Schottky groups . . . . . . . . . . . . . . . 19

${ }^{*}$ The first author gratefully acknowledges the support by the National Science Foundation. The second author appreciates the hospitality of the mathematics department of the University of Utah. 
6 Weak Proper Discontinuity and Infinite Dimension of $\widetilde{Q H}(G) 23$

7 Reducible nonpositively curved manifolds 25

8 Teichmuller space with Weil-Petersson metric 3

\section{Introduction}

Let $\Gamma$ be a group. A function $\phi: \Gamma \rightarrow \mathbb{R}$ is called a quasi-homomorphism provided

$$
\Delta(\phi):=\sup _{x, y \in \Gamma}|\phi(x y)-\phi(x)-\phi(y)|<\infty
$$

The vector space

$\widetilde{Q H}(\Gamma)=\{$ quasi-homomorphisms $\phi: \Gamma \rightarrow \mathbb{R}\} / \operatorname{Hom}(\Gamma, \mathbb{R})+\{$ bounded $\phi: \Gamma \rightarrow \mathbb{R}\}$

can be identified with the kernel $\operatorname{Ker}\left(H_{b}^{2}(\Gamma ; \mathbb{R}) \rightarrow H^{2}(\Gamma ; \mathbb{R})\right)$ from the bounded cohomology of $\Gamma$ to the ordinary cohomology.

A quasi-homomorphism $\phi$ is homogeneous if $\phi\left(g^{n}\right)=n \phi(g)$ for all $g \in \Gamma$ and $n \in \mathbb{Z}$. If we regard two quasi-homomorphisms $\phi_{1}, \phi_{2}$ as equivalent if $\phi_{1}-\phi_{2}$ is bounded, then each equivalence class contains a unique homogeneous representative. Note that homogeneous quasi-homomorphisms are constant on conjugacy classes and that

$$
\widetilde{Q H}(\Gamma)=\{\text { homogeneous quasi-homomorphisms } \phi: \Gamma \rightarrow \mathbb{R}\} / \operatorname{Hom}(\Gamma, \mathbb{R})
$$

There is a growing list of groups $\Gamma$ for which $\widetilde{Q H}(\Gamma)$ has been "computed". If $\Gamma$ is amenable then $\widetilde{Q H}(\Gamma)=0$. The celebrated work of BurgerMonod [8] 9] implies that $\widetilde{Q H}(\Gamma)=0$ for any irreducible lattice $\Gamma$ in a semi-simple Lie group $G$ with finite center. (Burger and Monod state their result in the language of algebraic groups. In particular, their assumption that the group is simply-connected refers to the associated group over $\mathbb{C}$. In fact, it suffices that the complex group has finite fundamental group.) On the other hand, non-elementary hyperbolic groups $\Gamma$, and indeed groups that act on $\delta$-hyperbolic spaces with a weak proper discontinuity condition (WPD) have infinite-dimensional $\widetilde{Q H}(\Gamma)[12,[13],[14,[6]$. That some condition on the action is needed can be seen by looking at an irreducible lattice $\Gamma$ in $\mathbb{H}^{2} \times \mathbb{H}^{2}$, which acts on $\mathbb{H}^{2}$ and has $\widetilde{Q H}(\Gamma)=0$ by the Burger-Monod theorem.

The constructions of quasi-homomorphisms in all these cases build on the construction of Brooks [7] for the case of free groups. In this paper we further 
extend the technology to the setting of $C A T(0)$ spaces. More precisely, the main theorem is the following. For definitions of rank 1 isometries and the WPD condition, see Sections 5 and 6 .

Main Theorem. Let $X$ be a $C A T(0)$ space and let $\Gamma$ be a group acting by isometries on $X$. Also suppose that $\Gamma$ is not virtually cyclic, that the action satisfies $W P D$, and that $\Gamma$ contains an element that acts as a rank 1 isometry. Then $\widetilde{Q H}(\Gamma)$ is infinite-dimensional.

In the Main Theorem we do not assume that $X$ is a proper, or even complete, metric space.

As an application, we prove the converse of the Burger-Monod result. The proof is based on the celebrated Rank Rigidity Theorem [1, Theorem C, Theorem III.3.4], [11, Theorem 9.4.1].

Rank Rigidity Theorem. Let $M$ be a complete Riemannian manifold of nonpositive curvature and finite volume. Consider the de Rham decomposition of the universal cover $\tilde{M}$ and assume that it has no Euclidean factor. Then one of the following holds.

(1) $\tilde{M}$ is a symmetric space of noncompact type and rank $>1$.

(2) Some deck transformation of $\tilde{M}$ is a rank 1 isometry.

(3) Some finite cover $M^{\prime}$ of $M$ splits as a nontrivial Riemannian product $M_{1}^{\prime} \times M_{2}^{\prime}$.

Our converse to the Burger-Monod theorem follows quickly.

Theorem 1.1. Let $M$ be a complete Riemannian manifold of nonpositive curvature and finite volume. Assume that $\Gamma=\pi_{1}(M)$ is finitely generated and does not contain a subgroup of finite index which is cyclic or a Cartesian product of two infinite groups. Then the universal cover $\tilde{M}$ is a higher rank symmetric space if and only if $\widetilde{Q H}(\Gamma)=0$. Otherwise, $\widetilde{Q H}(\Gamma)$ is infinitedimensional.

Proof. Consider the de Rham decomposition of $\tilde{M}$. One possibility is that $\tilde{M}$ is Euclidean space. Then $\Gamma$ is a Bieberbach group and therefore contains $\mathbb{Z}^{n}$ as a subgroup of finite index, contradicting our assumptions. If the de Rham decomposition contains a Euclidean factor and a non-Euclidean factor, then some finite cover $M^{\prime}$ of $M$ is diffeomorphic to the product $N \times T$ for a nonpositively curved manifold $N$ of finite volume and a torus $T$ [3. Theorem 1.9] (this uses that the group is finitely generated), again 
violating the assumptions. If there are no Euclidean factors, then we apply the Rank Rigidity Theorem. Possibility (3) is excluded by our assumption on $\Gamma$. If $\tilde{M}$ is a symmetric space of rank $>1$ the Burger-Monod theorem implies $\widetilde{Q H}(\Gamma)=0$, and if $\tilde{M}$ admits a rank 1 isometry the Main Theorem implies that $\widetilde{Q H}(\Gamma)$ is infinite-dimensional.

The condition $\widetilde{Q H}(\Gamma)=0$ can be cast, by the work of Bavard [4], in terms of stable commutator length. Recall that for $\gamma \in[\Gamma, \Gamma]$ the commutator length $c(\gamma)$ is the smallest $k \geq 0$ such that $\gamma$ can be expressed as a product of $k$ commutators. The stable commutator length is the limit

$$
c_{\infty}(\gamma)=\lim _{n \rightarrow \infty} \frac{c\left(\gamma^{n}\right)}{n}
$$

Bavard's theorem states that $\widetilde{Q H}(\Gamma)=0$ if and only if $c_{\infty}$ vanishes on $[\Gamma, \Gamma]$.

There are examples of groups $\Gamma$ such that $\widetilde{Q H}(\Gamma)$ is nonzero and finite dimensional. All known examples are constructed via central extensions as a variation of the following argument (see [15], [17]). Start with a group $\Gamma_{0}$ with $\widetilde{Q H}\left(\Gamma_{0}\right)=0$ and with $H^{2}\left(\Gamma_{0} ; \mathbb{R}\right) \neq 0$. Let $1 \rightarrow \mathbb{Z} \rightarrow \Gamma \rightarrow \Gamma_{0} \rightarrow 1$ be a central extension whose Euler class $e \in H^{2}\left(\Gamma_{0} ; \mathbb{R}\right)$ is nonzero and in the image of $H_{b}^{2}(\Gamma ; \mathbb{R}) \rightarrow H^{2}(\Gamma ; \mathbb{R})$. Then $H^{2}(\Gamma ; \mathbb{R}) \cong H^{2}\left(\Gamma_{0} ; \mathbb{R}\right) /\langle e\rangle$ while $H_{b}^{2}(\Gamma ; \mathbb{R}) \cong H_{b}^{2}\left(\Gamma_{0} ; \mathbb{R}\right)$, so $\widetilde{Q H}(\Gamma)$ is 1-dimensional.

Theorem 1.2. Suppose $M$ is a complete Riemannian manifold of nonpositive curvature and finite volume. If $\Gamma=\pi_{1}(M)$ is finitely generated then $\widetilde{Q H}(\Gamma)$ is either 0 or infinite-dimensional.

We will prove Theorem 1.2 in Section 7 In the special case when $\tilde{M}$ does not have Euclidean space as a de Rham factor, and no finite cover of $M$ is a nontrivial Riemannian product, the statement follows from Theorem 1.1 .

Another application is to the action of the mapping class group, $M C G(S)$, of a compact orientable surface $S$ on the Teichmüller space, $T(S)$, with WeilPetersson metric. We will show the following in Section 8 . This result was shown in [6] using the action of $M C G(S)$ on the curve complex.

Theorem 1.3. Let $S$ be a compact orientable surface, and $M C G(S)$ its mapping class group. Suppose $\Gamma<M C G(S)$ is not virtually cyclic and contains at least one pseudo-Anosov element. Then, $\widetilde{Q H}(\Gamma)$ is infinitedimensional. 
The present proof raises hopes that a similar theorem can be proved for subgroups of $\operatorname{Out}\left(F_{n}\right)$, since in that case there is no analog of the curve complex (which is presently known to be hyperbolic). Of course, there are still formidable obstacles, not the least of which is finding a suitable metric on Outer space.

With an eye towards Outer space, we have tried to axiomatize the properties of the space $X$ our method requires to show that $\widetilde{Q H}(\Gamma)$ is infinite dimensional. There are two axioms: (DD) and (FT) (see the next section). Both hold in $C A T(0)$ spaces as well as in $\delta$-hyperbolic spaces.

We list the two axioms in Section 2. In Section 3 we introduce the key concept of $B$-contracting segments and discuss the basic properties. In Section 4 we construct many quasi-homomorphisms in the situation when our axioms hold. The key is a certain "thick-thin" decomposition of a geodesic triangle (see the proof of Theorem 4.6). In Section 5 we introduce the concept of a rank 1 isometry and construct a "Schottky group" (a free group of isometries in which every nontrivial element is rank 1) starting with two independent rank 1 isometries. This is then used in Section 6 to show that the quasi-homomorphisms constructed in Section 4 span an infinite-dimensional vector space. Finally, Section 8 contains the verification of our axioms for the Weil-Petersson metric on Teichmüller space.

Acknowledgements. We wish to thank Ken Bromberg for pointing out that we should subdivide the ruled rectangles into two triangles in the proofs of Section 8. We also thank Werner Ballmann, Emmanuel Breuillard, Ursula Hamenstädt and Nicolas Monod for useful conversations and to Werner Ballmann for correcting a mistake in an earlier version of the manuscript. We are grateful to the referee for carefully reading the manuscript and suggesting we add Section 7 .

\section{Axioms}

Let $(X, d)$ be a geodesic metric space. Thus any two points $a, b \in X$ are connected by at least one geodesic. To simplify notation we will usually denote such a geodesic by $[a, b]$ even though it may not be unique. We will also usually denote by $|a-b|$ the distance $d(a, b)$. For the rest of the paper we will make the following assumptions on $X$.

For any geodesic segment $[a, b]$ and $x \in X$ denote by $\pi_{a b}(x)$ the (closed) set of points in $[a, b]$ that minimize the distance to $x$. We think of $\pi_{a b}(x)$ as the (multivalued) projection of $x$ to $[a, b]$. The constant $C>0$ is fixed.

The first axiom states that the projection coarsely decreases distances. 
(DD) For every $p \in \pi_{a b}(x)$ and every $p^{\prime} \in \pi_{a b}\left(x^{\prime}\right)$ we have

$$
\left|p-p^{\prime}\right|<\left|x-x^{\prime}\right|+C .
$$

Note that, in particular, the diameter of $\pi_{a b}(x)$ is $<C$.

Our second axiom on $(X, d)$ is the strong version of the Fellow Traveller Property.

(FT) Suppose $\left|a-a^{\prime}\right| \leq D,\left|b-b^{\prime}\right| \leq D$. Then $\left[a^{\prime}, b^{\prime}\right]$ is contained in the Hausdorff $C+D$-neighborhood of $[a, b]$.

In particular, any two geodesics from $a$ to $b$ are in each others' Hausdorff $C$-neighborhood.

Proposition 2.1. Every $C A T(0)$ space (or more generally, a geodesic metric space with a strictly convex distance function) as well as every $\delta$-hyperbolic space satisfies axioms (DD) and (FT).

Remark 2.2. The strong version of the Fellow Traveller Property (FT) is used only in the proof of Proposition 4.2. For the rest of the paper a weaker version (FT-) would suffice: $\left[a^{\prime}, b^{\prime}\right]$ is contained in the Hausdorff $F T(C, D)$ neighborhood for some function $F T:[0, \infty) \times[0, \infty) \rightarrow[0, \infty)$.

\section{$3 \quad B$-contracting segments}

From now on, we assume that $X$ is a $\mathrm{CAT}(0)$ space satisfying Axioms (DD) and (FT).

Definition 3.1. A geodesic segment $[a, b]$ is said to be $B$-contracting for $B>0$ if for every metric ball $K$ disjoint from $[a, b]$ the projection $\pi_{a b}(K)$ has diameter $<B$.

Lemma 3.2 (Subsegments contracting). There is $B^{\prime}>0$ (e.g. $B^{\prime}=B+$ $4 C+3$ works) such that every subsegment of a B-contracting segment is $B^{\prime}$-contracting.

Proof. Let $[a, b]$ be a $B$-contracting segment and let $K$ be a ball centered at $O \in X$ and disjoint from $[u, v] \subset[a, b]$. For concreteness, say $u$ is between $a$ and $v$. We will also assume that $d(u, v)>C$ for otherwise $[u, v]$ is $C+1$ contracting. There are two cases.

Case 1. $\pi_{a b}(O) \cap[u, v] \neq \emptyset$. Then $K$ is also disjoint from $[a, b]$ and so $\pi_{a b}(K)$ has diameter $<B$. Let $x \in K$. If $\pi_{a b}(x) \cap[u, v] \neq \emptyset$ then 
$\pi_{u v}(x)=\pi_{a b}(x) \cap[u, v]$ and if $\pi_{a b}(x) \cap[u, v]=\emptyset$ then $\pi_{a b}(x)$ is contained either in $[a, u]$ or in $[v, b]$ by Axiom (DD). Say $\pi_{a b}(x) \subset[a, u]$. Again by (DD), it follows that $\pi_{u v}(x)$ is contained in the $C$-neighborhood of $u$. (For suppose that $t \in \pi_{u v}(x)$ and $|t-u|>C$. Let $s \in[a, u]$ be the last point with $|s-x|=|t-x|$. Then both endpoints of $[s, t]$ are in $\pi_{s t}(x)$ and $|s-t|>C$.) If there are any such $x \in K$ then $u$ is in the smallest interval containing $\pi_{a b}(K)$, so it follows that in either case the diameter of $\pi_{u v}(K)$ is bounded above by $B+C$.

Case 2. $\pi_{a b}(O) \cap[u, v]=\emptyset$. Again, for concreteness, we may assume $\pi_{a b}(O) \subset[a, u]$. In this case $K$ may intersect $[a, b]$, so we construct a different ball.

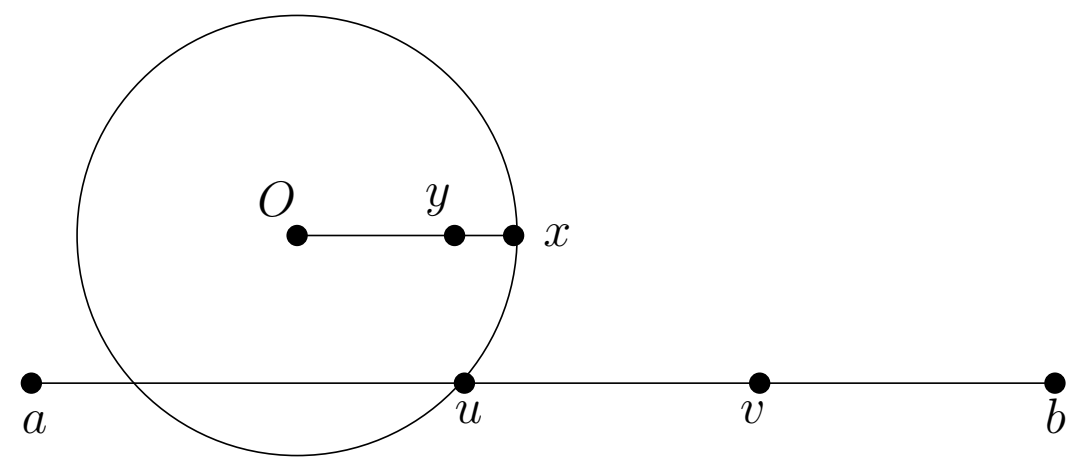

Figure 1: Lemma 3.2

Suppose that there is $x \in K$ such that $\pi_{u v}(x)$ is not contained in the $B+4 C+3$-neighborhood of $u$. It follows that $\pi_{a b}(x)$ is not contained in the $B+3 C+3$-neighborhood of $[a, u]$. By subdividing a segment $[O, x]$ into intervals of size $<1$ and using Axiom (DD) we find a point $y \in[O, x]$ such that $\pi_{a b}(y)$ is contained in the $C+1$-neighborhood of $u$. Let $K^{\prime}$ be the closed ball centered at $y$ with radius $d(y,[a, b])-1$. Thus by assumption $\pi_{a b}\left(K^{\prime}\right)$ has diameter less than $B$. Note that

$$
|y-u| \geq|O-u|-|O-y|=|O-u|-|O-x|+|y-x| \geq|y-x|
$$

(The last inequality follows from the fact that $u$ is outside $K$ while $x$ is inside.)

Now radius of $K^{\prime}$ is $d(y,[a, b])-1 \geq|y-u|-C-2$ and therefore $K^{\prime}$, even if it does not contain $x$, must contain a point at distance $\leq C+2$ from $x$. The projection of this point to $[a, b]$ is then, by (DD), not contained in the $B+3 C+3-(2 C+2)=B+C+1$-neighborhood of $[a, u]$. Since 
$\pi_{a b}(y)$ is contained in the $C+1$-neighborhood of $u$ it follows that $\pi_{a b}(K)$ has diameter $\geq B$, contradiction.

For clarity, we will not try to keep up with constants such as $B+4 C+3$ in Lemma 3.2, but rather we introduce the following function notation: $\Phi=\Phi(B, C)$ stands for a positive function which is monotonically increasing in each variable. Sometimes we decorate $\Phi$ with a subscript, and sometimes there are more than 2 arguments. When we want to refer to a $\Phi$-function from a previous lemma, we will indicate the number in the subscript, e.g. $\Phi_{3.2}(B, C)=B+4 C+3$ and $\Phi_{3.5}(B, C)=3 B+C+1$.

Lemma 3.3. Suppose $[x, y]$ and $[u, v]$ are geodesic segments such that $u \in$ $\pi_{u v}(x)$ and $v \in \pi_{u v}(y)$. Also suppose that the distance between $[x, y]$ and $[u, v]$ is larger than $|u-v|$. Then $[x, y]$ is contained in the union of 5 balls disjoint from $[u, v]$.

Proof. Let $[p, q]$ be a shortest geodesic segment connecting a point $p \in[x, y]$ with a point $q \in[u, v]$. Then

$$
|p-y| \leq|p-q|+|q-v|+|y-v|<2|p-q|+|y-v|
$$

so the open balls centered at $p, x, y$ with radius equal to the distance to $[u, v]$ leave at most two segments in $[x, y]$ uncovered, and these segments have length $<|p-q|$ so each can be covered by one more ball.

Corollary 3.4. Suppose $[x, y]$ and $[u, v]$ are geodesic segments such that $u \in \pi_{u v}(x)$ and $v \in \pi_{u v}(y)$. Also suppose that $[u, v]$ is $B$-contracting. Then either $|u-v|<\Phi(B, C)$ or the distance between $[x, y]$ and $[u, v]$ is $<\Phi(B, C)$.

Proof. By Lemma 3.2 there is $B^{\prime}=\Phi_{3.2}(B, C)$ such that every subsegment of $[u, v]$ is $B^{\prime}$-contracting. We are done if the distance $D$ between $[x, y]$ and $[u, v]$ is $\leq 5 B^{\prime}+2 C$ so suppose it is $>5 B^{\prime}+2 C$. Now pass to a subinterval $\left[u^{\prime}, v^{\prime}\right] \subset[u, v]$ of length $5 B^{\prime}+2 C$ whose distance to $[x, y]$ is also $D$. Further, pass to a subinterval $\left[u^{\prime \prime}, v^{\prime \prime}\right] \subset\left[u^{\prime}, v^{\prime}\right]$ by moving the endpoints at most $C$ to ensure that $u^{\prime \prime} \in \pi_{u^{\prime \prime} v^{\prime \prime}}(x)$ and $v^{\prime \prime} \in \pi_{u^{\prime \prime} v^{\prime \prime}}(y)$. The distance between $\left[u^{\prime \prime}, v^{\prime \prime}\right]$ and $[x, y]$ is $\geq D$ so Lemma 3.3 applies to $[x, y]$ and $\left[u^{\prime \prime}, v^{\prime \prime}\right]$ and shows that $\left|u^{\prime \prime}-v^{\prime \prime}\right|<5 B^{\prime}$, a contradiction.

Lemma 3.5 (Thin Triangles). Suppose $[a, b]$ is B-contracting and $b \in$ $\pi_{a b}(c)$. Then

$$
d(b,[a, c])<3 B+C+1
$$

In the proof we need the following. 
Lemma 3.6. Suppose $[a, b]$ is B-contracting and $b \in \pi_{a b}(c)$. Then

$$
|a-b|+|b-c| \geq|a-c| \geq|a-b|+|b-c|-B-C-1
$$

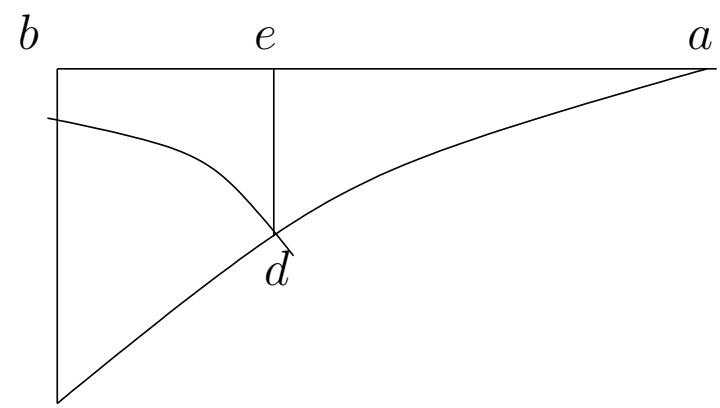

C

Figure 2: Lemma 3.5 and Lemma 3.6

Proof. Consider the ball centered at $c$ with radius $|b-c|-1$. By assumption, the projection of this ball to $[a, b]$ has diameter $<B$. Let $d$ be the point on $[c, a]$ at distance $|b-c|-1$ from $c$ (if $|b-c|<1$ there is nothing to prove). Denote by $e$ a point in $\pi_{a b}(d)$. Thus $|b-e|<B$. Now

$$
\begin{array}{r}
|a-c|-|b-c|=|c-d|+|d-a|-|b-c|=|b-c|-1+|d-a|-|b-c|= \\
|d-a|-1 \geq|e-a|-C-1=|a-b|-|b-e|-C-1>|a-b|-B-C-1
\end{array}
$$

where the first inequality comes from (DD).

Proof of Lemma 3.5. Let $q \in[a, c]$ be the point with $|c-q|=|c-b|$. We will argue that $|b-q|$ is bounded.

Let $r \in \pi_{a b}(q)$. Note that $|b-r|<B$. Subtracting $|b-c|=|c-q|$ from $|a-c| \leq|a-b|+|b-c|$ yields $|a-q| \leq|a-b|$ and so $|a-q|<|a-r|+B$. On the other hand, Lemma 3.6 implies that $|a-q|>|a-r|+|r-q|-B-C-1$. Comparing these inequalities we deduce $|r-q|<2 B+C+1$. It follows that $|b-q| \leq|b-r|+|r-q|<3 B+C+1$.

Lemma 3.7. Let $[a, b]$ be a B-contracting segment and assume $|b-c| \leq D$. Let $x \in X$ and denote by $x_{1}, x_{2}$ projections of $x$ onto $[a, b],[a, c]$ respectively (i.e. $x_{1} \in \pi_{a b}(x), x_{2} \in \pi_{a c}(x)$ ). Let $x_{3}$ be a projection of $x_{2}$ onto $[a, b]$. Then $\left|x_{1}-x_{3}\right| \leq \Phi(B, C, D)$. 


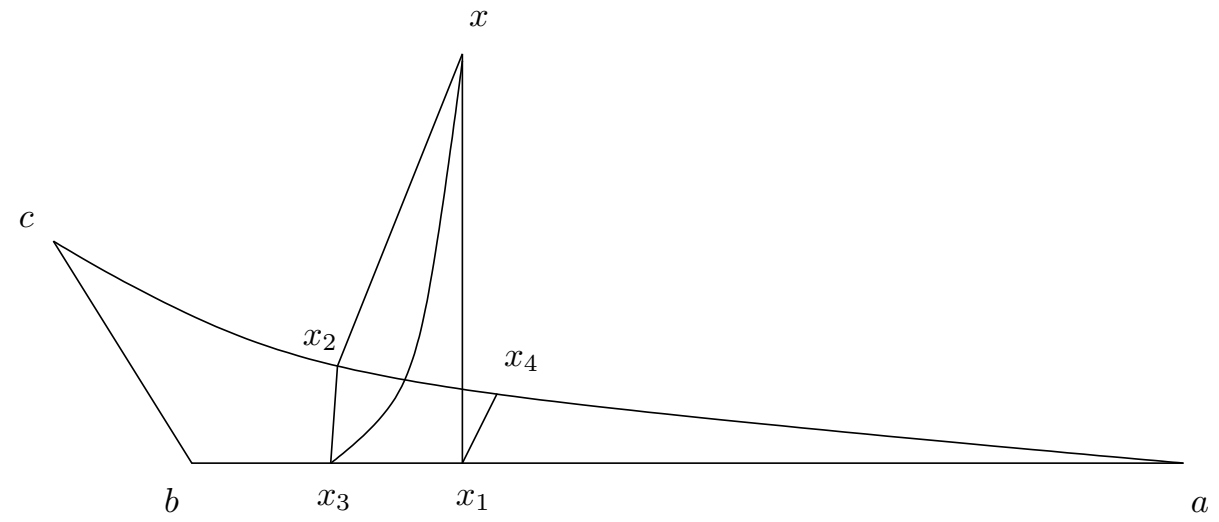

Figure 3: Lemma 3.7

Proof. Let $x_{4}$ be a projection of $x_{1}$ onto $[a, c]$. By Lemma 3.6

$$
\left|x_{3}-x\right|>\left|x_{3}-x_{1}\right|+\left|x_{1}-x\right|-\Phi[\overline{3.6}(B, C)
$$

By Axiom (FT) we have

$\left|x-x_{2}\right| \geq\left|x-x_{3}\right|-\left|x_{2}-x_{3}\right|>\left|x-x_{1}\right|+\left|x_{1}-x_{3}\right|-\Phi_{\overline{3.6}}(B, C)-C-D$

Again by (FT)

$$
\left|x-x_{4}\right| \leq\left|x-x_{1}\right|+\left|x_{1}-x_{4}\right| \leq\left|x-x_{1}\right|+C+D
$$

Since $\left|x-x_{4}\right| \geq\left|x-x_{2}\right|$ we deduce

$$
\left|x-x_{1}\right|+C+D>\left|x-x_{1}\right|+\left|x_{1}-x_{3}\right|-\Phi_{[3.6}(B, C)-C-D
$$

and the claim follows.

Lemma 3.8 (Stability). If $[a, b]$ is B-contracting, $\left|a-a^{\prime}\right|,\left|b-b^{\prime}\right| \leq D$ then $\left[a^{\prime}, b^{\prime}\right]$ is $\Phi(B, C, D)$-contracting.

Proof. It suffices to prove this in the special case $a=a^{\prime}$, for the general case can be obtained by applying the special case twice.

Consider a ball disjoint from $\left[a, b^{\prime}\right]$. Suppose the projection to $\left[a, b^{\prime}\right]$ has diameter $K$. After shrinking the radius by $C+D$ the ball is also disjoint from $[a, b]$, its projection $P$ to $\left[a, b^{\prime}\right]$ has diameter $\geq K-(C+D)-2 C$, and its projection $Q$ to $[a, b]$ has diameter $<B$. Every point of $P$ is within $C+D$ 
of its projection to $[a, b]$, which in turn is within $\Phi \overline{3.7}(B, C, D)$ of a point in $Q$. Thus $P$ is contained in the $C+D+\Phi_{3.7}(B, C, D)$-neighborhood of $Q$ and also in any ball of radius $C+D+\Phi \overline{3.7}(B, C, D)+B$ centered at a point of $Q$. It follows that $K<2(C+D+\Phi \underline{3.7}(B, C, D)+B)+C+D+2 C$.

\section{Quasi-homomorphisms}

In this section we construct many quasi-homomorphisms $\Gamma \rightarrow \mathbb{R}$ for a group $\Gamma$ that acts isometrically on the CAT(0) space $X$ satisfying (DD) and (FT). In Section 6 we will see that many of them are nontrivial (and linearly independent) under suitable assumptions on the action.

Proposition 4.1. Let $[a, b]$ be a B-contracting segment and $[p, q]$ an arbitrary segment. Assume that the distance to $[p, q]$ along $[a, b]$ is minimized at $a$ and is equal to $d \geq 1$. Then

$$
d(b,[p, q])-d(a,[p, q]) \geq\left(1-\frac{B}{d}\right)|a-b|-\Phi(B, C) .
$$

Thus when $d>>B$ the segment $[a, b]$ is, for the most part, moving "orthogonally" to $[p, q]$.

Proof. Let $r$ be a projection of $b$ to $[p, q]$, let $s$ be a projection of $r$ to $[a, b]$ and let $t \in[p, q]$ be such that $|a-t|=d$. Subdivide $[t, r]$ into the smallest possible number, say $K$, of subintervals so that each has length $<d$. Therefore each subinterval has the projection to $[a, b]$ of diameter $<B$ and we conclude that $|a-s|<K B$. We have

$$
\begin{array}{r}
d(b,[p, q])=|b-r| \geq|b-s|+|s-r|-\Phi_{[3.6}(B, C) \geq \\
|b-s|+d-\Phi_{[3.6}(B, C) \geq|a-b|-K B+d-\Phi_{[3.6}(B, C)
\end{array}
$$

Since $t$ and $r$ are projections of $a$ and $b$, (DD) implies that

$$
|a-b| \geq|t-r|-C \geq(K-1) d-C
$$

and we deduce that $K \leq 1+\frac{|a-b|+C}{d}$. Therefore

$d(b,[p, q])-d>|a-b|-K B-\Phi_{[\overline{3.6}}(B, C) \geq|a-b|-\left(1+\frac{|a-b|+C}{d}\right) B-\Phi_{\left[{ }_{3.6}\right.}(B, C)$

and the claim follows. 


\subsection{Expressways}

Assume a group $\Gamma$ acts on $X$ by isometries. Fix a collection $\mathcal{E}$ of geodesic segments in $X$ (thought of as expressways), subject to the following axioms. The constants $L$ and $B$ are fixed ( $L$ will be chosen large using Proposition 4.2).

- $\mathcal{E}$ is $\Gamma$-equivariant,

- each $\sigma \in \mathcal{E}$ is $B$-contracting,

- each $\sigma \in \mathcal{E}$ has length $L$.

To each piecewise geodesic path $\alpha=\left[a_{0}, a_{1}\right] \cup\left[a_{1}, a_{2}\right] \cup \cdots \cup\left[a_{k-1}, a_{k}\right]$ in $X$ we will assign modified length, equal to $m l(\alpha)=\sum_{i=1}^{k}\left|a_{i-1}-a_{i}\right|-e$ where $e$ is the number of segments $\left[a_{i-1}, a_{i}\right]$ which are expressways. We can think of modified length as time needed to travel along the path, provided we travel with speed 1 on regular roads and with fixed speed $>1$ on expressways.

We will call a piecewise geodesic path admissible if for any two consecutive geodesic segments $\left[a_{i-1}, a_{i}\right] \cup\left[a_{i}, a_{i+1}\right]$, at least one is an expressway. Note that if neither is an expressway, then replacing $\left[a_{i-1}, a_{i}\right] \cup\left[a_{i}, a_{i+1}\right]$ by $\left[a_{i-1}, a_{i+1}\right]$ does not increase modified length, and a repeated application of this operation converts the path to an admissible path.

Now fix a (long) geodesic segment $[p, q]$. In the next Proposition we will study admissible paths that join $p$ to $q$ and minimize modified length (with respect to a fixed collection $\mathcal{E}$ of expressways). When $X$ is a proper metric space it is easy to see that such minimizers exist; in general we study admissible paths whose modified length is close to minimal. We will show that such paths are necessarily contained in a Hausdorff neighborhood of $[p, q]$.

Proposition 4.2. There is a function $\Phi(B, C)$ with the following property. Assume that expressways have length $L>\Phi(B, C)$. Then for every segment $[p, q]$ every admissible path that minimizes modified distance from $p$ to $q$ is contained in the Hausdorff $\Phi(B, C)$-neighborhood of $[p, q]$. The same is true (even if no minimizing paths exist) for each admissible path within 1 of the infimum.

In the proof we need the following lemma.

Lemma 4.3. Let $\left[t, t^{\prime}\right]$ be a geodesic segment in $X$ and let $[t, y] \cup[y, z] \cup\left[z, t^{\prime}\right]$ be a piecewise geodesic joining $t$ and $t^{\prime}$. Also assume that every subsegment of $[t, y]$ and of $\left[z, t^{\prime}\right]$ is $B^{\prime}$-contracting. Then either 
(1) $|t-y|+|y-z|+\left|z-t^{\prime}\right|-\left|t-t^{\prime}\right|>3$, or

(2) $[t, y] \cup[y, z] \cup\left[z, t^{\prime}\right]$ is contained in the $\Phi\left(B^{\prime}, C\right)$-neighborhood of $\left[t, t^{\prime}\right]$.

Proof. We may assume that one of $[t, y]$ or $\left[z, t^{\prime}\right]$, say $\left[z, t^{\prime}\right]$, is degenerate (a point), since the general case follows by applying the special case twice.

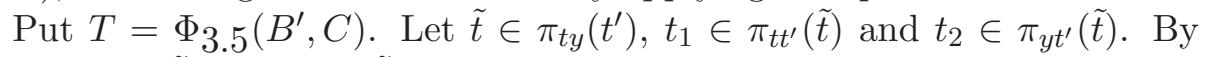
Lemma 3.5, $\left|\tilde{t}-t_{1}\right|<T,\left|\tilde{t}-t_{2}\right|<T$.

The "savings" $|t-y|+\left|y-t^{\prime}\right|-\left|t-t^{\prime}\right|$ is equal to $\left(|t-\tilde{t}|-\left|t-t_{1}\right|\right)+\left(\mid t_{2}-\right.$ $\left.t^{\prime}|-| t_{1}-t^{\prime} \mid\right)+\left(|\tilde{t}-y|+\left|y-t_{2}\right|\right)$, which is at least $-T-2 T+(2|\tilde{t}-y|-T)$. Therefore, if (1) does not hold, then this number is less than 3 , so that $|\tilde{t}-y|<2 T+\frac{3}{2}$. It follows that $[t, y]$ is within distance $T+C+\left(2 T+\frac{3}{2}\right)$ from $\left[t, t^{\prime}\right]$. Also, $\left[y, t^{\prime}\right]$ is within distance $2 T+C+\left(2 T+\frac{3}{2}\right)+T$ from $\left[t, t^{\prime}\right]$. Put $\Phi\left(B^{\prime}, C\right)=5 T+C+\frac{3}{2}$, then (2) holds.

Proof of Proposition 4.2. Fix a (nearly) minimizing admissible path $\alpha=$ $\left[a_{0}, a_{1}\right] \cup\left[a_{1}, a_{2}\right] \cup \cdots \cup\left[a_{k-1}, a_{k}\right]$ from $p=a_{0}$ to $q=a_{k}$. For each expressway $\sigma=\left[a_{i}, a_{i+1}\right]$ in $\alpha$ let $\Delta(\sigma)$ denote the distance between $\sigma$ and $[p, q]$. Also, let $B^{\prime}=\Phi[3.2(B, C)$ so that subsegments of expressways in $\alpha$ are $B^{\prime}$-contracting.

There are now two cases.

Case 1. Maximal $\Delta(\sigma)$ is $\geq \max \left(1,2 B^{\prime}\right)$. Say the maximum is realized on the expressway $\sigma=\left[a_{i}, a_{i+1}\right]$ and let $t \in \sigma, \hat{t} \in[p, q]$ be such that $|t-\hat{t}|=$ $\Delta(\sigma)$. For concreteness, say $\left|t-a_{i+1}\right| \geq\left|t-a_{i}\right|$. Then consider the subpath $\left[a_{i}, a_{i+1}\right] \cup\left[a_{i+1}, a_{i+2}\right] \cup\left[a_{i+2}, a_{i+3}\right]=[x, y] \cup[y, z] \cup[z, w]$ (we certainly cannot have $i+1=k$; if $i+2=k$ then $\left[a_{i+2}, a_{i+3}\right]=[z, w]$ is degenerate). Let $t^{\prime} \in$ $[z, w]$ be a point closest to $[p, q]$. By the choice of $\sigma, d(t,[p, q]) \geq d\left(t^{\prime},[p, q]\right)$ and by Axiom (FT) $\left[t, t^{\prime}\right]$ is contained in the $C+\Delta(\sigma)$-neighborhood of $[p, q]$. Now we may apply Lemma 4.3. If (1) holds, then replacing the portion $[x, y] \cup[y, z] \cup[z, w]$ of $\alpha$ by $[x, t] \cup\left[t, t^{\prime}\right] \cup\left[t^{\prime}, w\right]$ results in a path $\alpha^{\prime}$ with modified length $m l\left(\alpha^{\prime}\right)<m l(\alpha)-1$. The path $\alpha^{\prime}$ may not be admissible, but we may replace it by an admissible path without increasing modified length. Thus $\alpha$ wasn't within 1 of the infimum. Therefore, (2) must hold and in particular $y$ is in the $d(t,[p, q])+C+\Phi_{4.3}\left(B^{\prime}, C\right)$-neighborhood of $[p, q]$. It follows that the distance function to $[p, q]$ has variation $\leq C+\Phi_{4.3}\left(B^{\prime}, C\right)$ along $[t, y]$. But this variation is at least $\frac{1}{2}|t-y|-\Phi_{4.1}\left(B^{\prime}, C\right)$ and we conclude

$$
L=|x-y| \leq 2|t-y| \leq 4\left(\Phi \overline{4.1}\left(B^{\prime}, C\right)+C+\Phi \overline{4.3}\left(B^{\prime}, C\right)\right)
$$

Case 2. Every expressway in $\alpha$ intersects the Hausdorff $\max \left(1,2 B^{\prime}\right)$-neighborhood of $[p, q]$. For each such expressway $\left[a_{i}, a_{i+1}\right]$ let $\left[s_{i}, t_{i}\right]$ be the maximal subin- 
terval with $d\left(s_{i},[p, q]\right) \leq \max \left(1,2 B^{\prime}\right)$ and $d\left(t_{i},[p, q]\right) \leq \max \left(1,2 B^{\prime}\right)$. Let $D_{i}$ be the length of the longer of the two complementary components (one or both of these might be empty; the length of the empty set is 0). Arguing exactly as in Case 1 , with $[t, y]=\left[t_{i}, a_{i+1}\right]$ or $[t, y]=\left[s_{i}, a_{i}\right]$, we conclude

$$
D_{i} \leq 2\left(\Phi_{4.1}\left(B^{\prime}, C\right)+\Phi_{4.3}\left(B^{\prime}, C\right)\right)
$$

(or else $\alpha$ is not within 1 of infimum). Therefore, $\alpha$ is in the Hausdorff $2\left(\Phi_{4.1}\left(B^{\prime}, C\right)+\Phi_{4.3}\left(B^{\prime}, C\right)\right)+\max \left(1,2 B^{\prime}\right)$-neighborhood of $[p, q]$.

For the rest of this section we will use the following notation. Let $D=$ $\Phi_{4.2}(B, C), S=\Phi \overline{3.8}(B, C, D), S^{\prime}=\Phi_{3.2}(S, C)$, and $T=\Phi_{3.4}\left(S^{\prime}, C\right)+D$.

Lemma 4.4. Every $B$-contracting segment $[x, y]$ of length $\geq \Phi(B, C)$ contained in the $D$-neighborhood of $[p, q]$ contains a point whose distance from $[p, r] \cup[r, q]$ is $<T$ for any $r \in X$.

Proof. Let $u$ be a projection of $x$ and $v$ a projection of $y$ to $[p, q]$. Then $[u, v]$ is $S$-contracting and every subsegment is $S^{\prime}$-contracting. Say a projection of $r$ to $[u, v]$ is closer to $v$ than to $u$. Assuming $|x-y|$ is sufficiently big, it follows that some point of $[u, v]$ is within $\Phi_{[3.4}\left(S^{\prime}, C\right)$ of $[p, r]$.

\subsection{Construction of quasi-homomorphisms}

Now assume that a group $\Gamma$ acts on $X$ by isometries. Fix a constant $L$ (in the definition of $\mathcal{E}$ ) to satisfy the assumption in Proposition 4.2, Let $\mathcal{E}$ be a collection of expressways. For $a, b \in X$ denote by

$$
\lambda(a, b)=\lambda_{\mathcal{E}}(a, b)
$$

the infimum of modified lengths of admissible piecewise geodesic paths from $a$ to $b$.

Proposition 4.5. $\quad \lambda(a, b) \leq|a-b|$.

- $\left|\lambda(a, b)-\lambda\left(a^{\prime}, b^{\prime}\right)\right| \leq\left|a-a^{\prime}\right|+\left|b-b^{\prime}\right|$.

- $\lambda(g a, g b)=\lambda(a, b)$ for any $g \in \Gamma$.

- If there are no expressways in the $D$-neighborhood of $[a, b]$ then $\lambda(a, b)=$ $|a-b|$.

- $\lambda(a, c) \leq \lambda(a, b)+\lambda(b, c)$ and if $b \in[a, c]$ then also $\lambda(a, c)>\lambda(a, b)+$ $\lambda(b, c)-2 D-1$. 
Proof. The first three statements follow immediately from the definition, the fourth one follows from Proposition 4.2. The first part of the last one is obvious, while the second part also follows from Proposition 4.2 by breaking up the (nearly) minimizing path from $a$ to $c$ at a point near $b$.

Now fix a basepoint $x_{0} \in X$ and a $B$-contracting segment $\sigma$. Let $\mathcal{E}$ consist of all translates of $\sigma$ by $\Gamma$. Define

$$
\phi_{\sigma}: \Gamma \rightarrow \mathbb{R}
$$

via

$$
\phi_{\sigma}(g)=\lambda\left(g\left(x_{0}\right), x_{0}\right)-\lambda\left(x_{0}, g\left(x_{0}\right)\right)
$$

Theorem 4.6. If $|\sigma|>\Phi(B, C)$ then $\phi_{\sigma}$ is a quasi-homomorphism, i.e.

$$
\left|\phi_{\sigma}\left(g g^{\prime}\right)-\phi_{\sigma}(g)-\phi_{\sigma}\left(g^{\prime}\right)\right|
$$

is uniformly bounded over all $g, g^{\prime} \in \Gamma$ (by a function of $B$ and $C$ ).

Proof. Consider the triangle with vertices $x_{0}, g\left(x_{0}\right), g g^{\prime}\left(x_{0}\right)$. We have

$$
\begin{aligned}
& \phi_{\sigma}(g)=\lambda\left(g\left(x_{0}\right), x_{0}\right)-\lambda\left(x_{0}, g\left(x_{0}\right)\right) \\
& \phi_{\sigma}\left(g^{\prime}\right)=\lambda\left(g g^{\prime}\left(x_{0}\right), g^{\prime}\left(x_{0}\right)\right)-\lambda\left(g^{\prime}\left(x_{0}\right), g g^{\prime}\left(x_{0}\right)\right) \\
& \phi_{\sigma}\left(g g^{\prime}\right)=\lambda\left(g g^{\prime}\left(x_{0}\right), x_{0}\right)-\lambda\left(x_{0}, g g^{\prime}\left(x_{0}\right)\right)
\end{aligned}
$$

Now consider the following "thick-thin" decomposition of the triangle. Each side is subdivided into 3 subintervals (the case when one or more middle intervals is degenerate is easier and is left to the reader). Figure 4.2 indicates the naming of the subdivision points. The point $a_{1}$ is chosen to be the last point in $\left[x_{0}, g\left(x_{0}\right)\right]$ whose distance to $\left[x_{0}, g g^{\prime}\left(x_{0}\right)\right]$ is $\leq T+D$, and similarly for the other 5 subdivision points.

Note that $\left|a_{1}-a_{2}\right| \leq C+T+D+2 T+2 D$ and similarly for the other two pairs. (To see this, let $t$ be the projection of $a_{2}$ onto $\left[x_{0}, g\left(x_{0}\right)\right]$. Then $t \in\left[x_{0}, a_{1}\right]$ by the choice of $a_{1}$. We will argue that $\left|t-a_{1}\right| \leq C+T+D+T+D$ and hence $\left|a_{1}-a_{2}\right| \leq C+T+D+2 T+2 D$. Let $s$ be a projection of $a_{1}$ onto $\left[x_{0}, g g^{\prime}\left(x_{0}\right)\right]$ (hence $s \in\left[x_{0}, a_{2}\right]$ ). By Axiom (FT) applied to $\left[x_{0}, a_{2}\right]$ and $\left[x_{0}, t\right]$ we see that $s$ is at distance $\leq C+T+D$ from some point $r \in\left[x_{0}, t\right]$. Thus $\left|r-a_{1}\right| \leq|r-s|+\left|s-a_{1}\right| \leq C+T+D+T+D$ and since $t \in\left[r, a_{1}\right]$ the claim is proved.)

Now replace each of the 6 terms above by the sum of 3 terms using the subdivision, e.g.

$$
\lambda\left(g\left(x_{0}\right), x_{0}\right)
$$




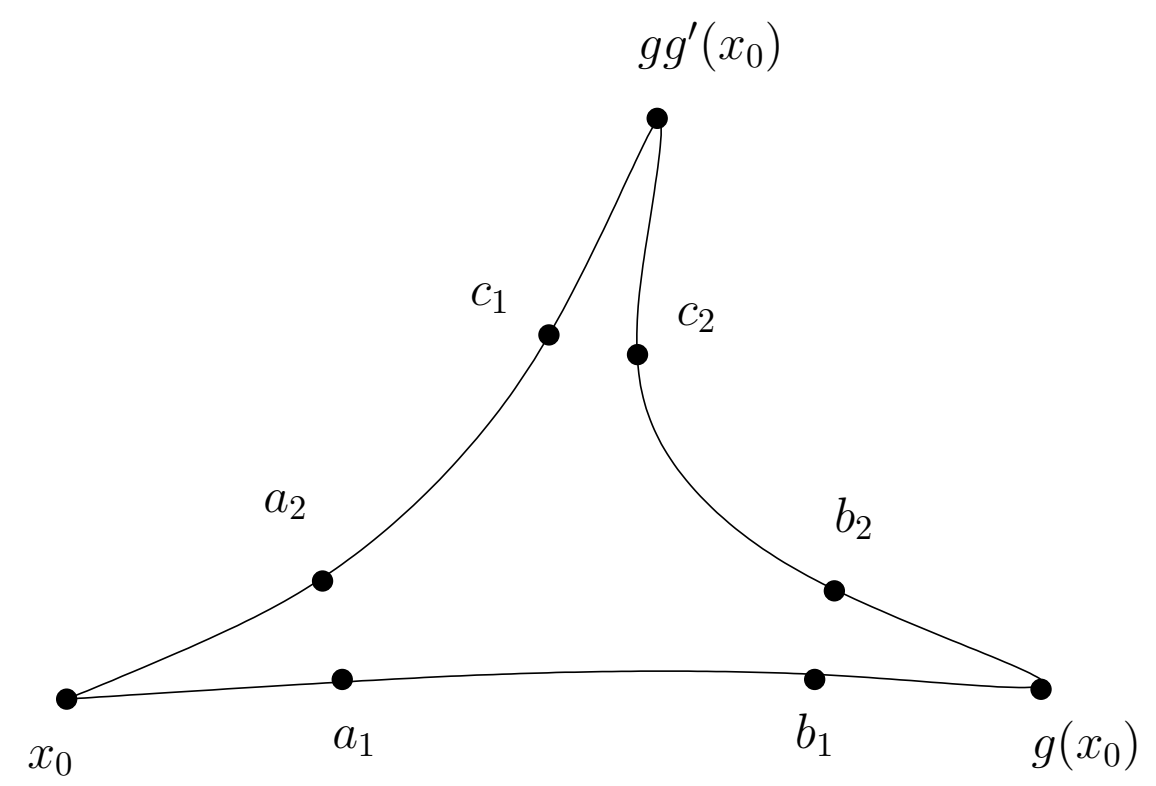

Figure 4: Thick-thin decomposition

by the sum

$$
\lambda\left(g\left(x_{0}\right), b_{1}\right)+\lambda\left(b_{1}, a_{1}\right)+\lambda\left(a_{1}, x_{0}\right) .
$$

Each such replacement introduces an error that does not exceed $4 D+2$ by Proposition 4.5. Then consider the sum $\phi_{\sigma}\left(g g^{\prime}\right)-\phi_{\sigma}(g)-\phi_{\sigma}\left(g^{\prime}\right)$. The terms such as $\lambda\left(a_{1}, b_{1}\right)-\lambda\left(b_{1}, a_{1}\right)$ cancel in pairs since they equal the distance $\left|a_{1}-b_{1}\right|$ (by Proposition 4.5 and Lemma 4.4). To see it, assume that $[x, y]$ is an expressway contained in the $D$-neighborhood of $\left[a_{1}, b_{1}\right]$. By Lemma 4.4 with $x_{0}=p, g\left(x_{0}\right)=q$ and $g g^{\prime}\left(x_{0}\right)=r$, there is a point $s$ in $[x, y]$ at distance $<T$ of $\left[x_{0}, g g^{\prime}\left(x_{0}\right)\right]$ or $\left[g\left(x_{0}\right), g g^{\prime}\left(x_{0}\right)\right]$, say $\left[x_{0}, g g^{\prime}\left(x_{0}\right)\right]$. There is a point $s^{\prime}$ in $\left[a_{1}, b_{1}\right]$ within $D$ of $s$. But then $s^{\prime}$ is at distance $<D+T$ of $\left[x_{0}, g g^{\prime}\left(x_{0}\right)\right]$, contradicting the choice of $a_{1}$. The terms such as $\lambda\left(x_{0}, a_{1}\right)-\lambda\left(x_{0}, a_{2}\right)$ also cancel, up to a bounded error by the above remark. At the end, all terms cancel and the total error is bounded as a function of $B$ and $C$.

\section{$5 \quad$ Rank 1 isometries}

We say that two subsets of a CAT(0) space $X$ are $B$-Hausdorff equivalent if each is contained in the $B$-Hausdorff neighborhood of the other. We continue to assume that $X$ satisfies Axioms (DD) and (FT). 
Definition 5.1. Let $g: X \rightarrow X$ be an isometry and let $x_{0} \in X$ be a basepoint. Denote $x_{n}=g^{n}\left(x_{0}\right)$. We say that $g$ is rank 1 if $\left|x_{n}-x_{0}\right| \rightarrow \infty$ as $n \rightarrow \infty$ and there exists $B>0$ such that for every $n>0$

- some geodesic $\left[x_{0}, x_{n}\right]$ is $B$-Hausdorff equivalent to $\left\{x_{0}, x_{1}, \cdots, x_{n}\right\}$, and

- some geodesic $\left[x_{0}, x_{n}\right]$ is $B$-contracting.

Using Axiom (FT) and Lemma 3.8 it is easy to see that the notion does not depend on the choice of the basepoint or the geodesics. It is also easy to see that there is $\epsilon>0$ such that $\left|x_{0}-x_{n}\right| \geq n \epsilon$ for all $n>0$.

Proposition 5.2. If $X$ is a $\delta$-hyperbolic space then every hyperbolic isometry $X \rightarrow X$ is rank 1 .

\subsection{Characterization when $X$ is a proper $C A T(0)$ space}

Ballmann and Brin 2 defined rank 1 isometries in the case when $X$ is a proper $C A T(0)$ space, namely, a hyperbolic isometry $g: X \rightarrow X$ is rank 1 if some (equivalently every) axis of $g$ fails to bound a half-flat.

To reconcile the two definitions, we show they are equivalent in this situation. We will need the following statement from the work of Ballmann, see the proof of Lemma III.3.3 in [1]. By $\bar{X}$ denote the usual visual compactification of $X$.

Proposition 5.3. Let $\ell$ be an axis of $g$ and assume that $g$ is rank 1. Let $\left\{x_{n}\right\}_{n}$ be a sequence of points in $X$ such that the projections of $x_{n}$ to $\ell$ leave every compact set. Then the accumulation set of the sequence $\left\{x_{n}\right\}$ in $\bar{X}$ is contained in the set of two endpoints of $\ell$.

Theorem 5.4. Let $X$ be a proper $C A T(0)$ space. Let $g: X \rightarrow X$ be a hyperbolic isometry with axis $\ell$. Then $\ell$ is $B$-contracting for some $B$ if and only if $\ell$ fails to bound a half-flat.

Proof. If $\ell$ bounds a half-flat then $\ell$ is clearly not $B$-contracting for any $B$.

Now assume that $\ell$ is not $B$-contracting for any $B$. Then there is a sequence of balls in the complement of $\ell$ whose projections to $\ell$ are larger and larger intervals. Denote by $\left\{z_{n}\right\}$ the sequence of centers of the balls. We may assume that the projection of $z_{n}$ to $\ell$ is contained in a fixed closed interval $J$ (a fundamental domain for $g: \ell \rightarrow \ell$ ). Let $x_{n}$ be a point in the $n$th ball chosen so that the sequence of projections of the $x_{n}$ 's to $\ell$ leave every compact set. By Proposition 5.3. after passing to a subsequence we 
may assume that the sequence $\left\{x_{n}\right\}$ converges to an endpoint $t \in \bar{X}$ of $\ell$, and also that the sequence $\left\{z_{n}\right\}$ converges to a point $\bar{z} \in \bar{X}$.

Moreover, we may assume that there exists some constant $r>0$ such that $d\left(x_{n}, \ell\right) \leq r$ for all $n$. This is because, otherwise, we can retake $x_{n}$ to satisfy this. Indeed, since $\ell$ does not bound a flat half plane, it does not bound a flat strip of width, say, $r>0$, since $X$ is proper. Let $Z_{n} \in J$ be the projection of $z_{n}$ to $\ell$. Let $x_{n}^{\prime}$ be the first point of the geodesic from $x_{n}$ to $Z_{n}$ to be contained in the $r$-neighborhood of $\ell$. (If $x_{n}$ is contained in the $r$-neighborhood, then $x_{n}^{\prime}=x_{n}$.) Then by [1, Lemma 3.1] combined with [1, Lemma 3.3], the projections of the $x_{n}^{\prime}$ 's to $\ell$ leave every compact set. We keep denoting those points by $x_{n}$ in the rest.

Consider the sequence of functions $f_{n}: X \rightarrow \mathbb{R}$ given by

$$
f_{n}(y)=\left|z_{n}-y\right|-d\left(z_{n}, \ell\right)
$$

This sequence converges to a Busemann function $f: X \rightarrow \mathbb{R}$ centered at $\bar{z}$. The horoball $f^{-1}(-\infty, 0]$ is convex, it includes a point of $J \subset \ell$, and its closure in $\bar{X}$ includes $t$. It follows that the ray $R$ of $\ell$ joining a point of $J$ with $t$ is contained in this horoball. (Indeed, $f_{n} \leq 0$ on $\left[x_{n}, Z_{n}\right]$. Since $x_{n}$ is contained in the $r$-neighborhood of $\ell$ and $Z_{n} \in J$, the sequence $\left[x_{n}, Z_{n}\right]$ converges to $R$. Thus $f \leq 0$ on $R$.) Moreover, each function $f_{n}$ is 1 -Lipschitz and non-negative on $\ell$, so the same holds for $f$. In particular, $f$ is identically 0 on the ray $R$.

Let $p \in R$ and consider the ray $l_{p}$ joining $p$ with $\bar{z}$. If we isometrically identify $l_{p}=[0, \infty)$ then $f(y)=-y$ for $y \in l_{p}$. It follows that the projection of $l_{p}$ onto $\ell$ is the point $p$.

Now take $p, q \in R$ and consider the function $g: l_{p}=[0, \infty) \rightarrow \mathbb{R}$ given by

$$
g(y)=d\left(y, l_{q}\right)
$$

This function is non-increasing because this is true for the analogous function defined with respect to two radial line segments sharing their terminal endpoints. We also have that $g(y) \geq|p-q|$ since the projection to $\ell$ doesn't increase distances and $l_{p}$ and $l_{q}$ project to $p, q$ respectively. It follows that $g$ is a constant function and hence $l_{p}$ and $l_{q}$ cobound a flat half-strip. In this way we can construct arbitrarily wide half-strips by taking $|p-q|$ large.

Now using the properness of $X$, a standard limiting argument applied to the translates of these half-strips by the powers of $g$ produces a half-flat bounded by $\ell$. 


\subsection{Schottky groups}

Lemma 5.5. Suppose $\left|q-q^{\prime}\right| \leq D, s \in[x, q], s^{\prime} \in\left[x, q^{\prime}\right]$. Then either $d\left(s,\left[s^{\prime}, q^{\prime}\right]\right) \leq \Phi(C, D)$ or $d\left(s^{\prime},[s, q]\right) \leq \Phi(C, D)$.

Proof. Let $r$ be a projection of $s^{\prime}$ to $[x, q]$ and $r^{\prime}$ a projection of $s$ to $\left[x, q^{\prime}\right]$. If $r^{\prime} \in\left[s^{\prime}, q^{\prime}\right]$ or if $r \in[s, q]$ we are done by Axiom (FT) $\left(\left|r-s^{\prime}\right|\right.$ and $\left|r^{\prime}-s\right|$ are bounded), so let us suppose that $r^{\prime} \in\left[x, s^{\prime}\right]$ and $r \in[x, s]$. Now apply (FT) to $[x, r]$ and $\left[x, s^{\prime}\right]$ to deduce that $d\left(r^{\prime},[x, r]\right)$ is bounded. Thus $|r-s|$ is bounded and so is $\left|s-s^{\prime}\right|$.

Lemma 5.6. Let $\sigma, \sigma^{\prime}$ be two B-contracting segments. Suppose that the minimal distance between $\sigma$ and $\sigma^{\prime}$ is $D$. Let $x \in X$ be an arbitrary point and let $p, p^{\prime}$ be projections of $x$ to $\sigma, \sigma^{\prime}$ respectively. Then either $d\left(p, \sigma^{\prime}\right) \leq$ $\Phi(B, C, D)$ or $d\left(p^{\prime}, \sigma\right) \leq \Phi(B, C, D)$.

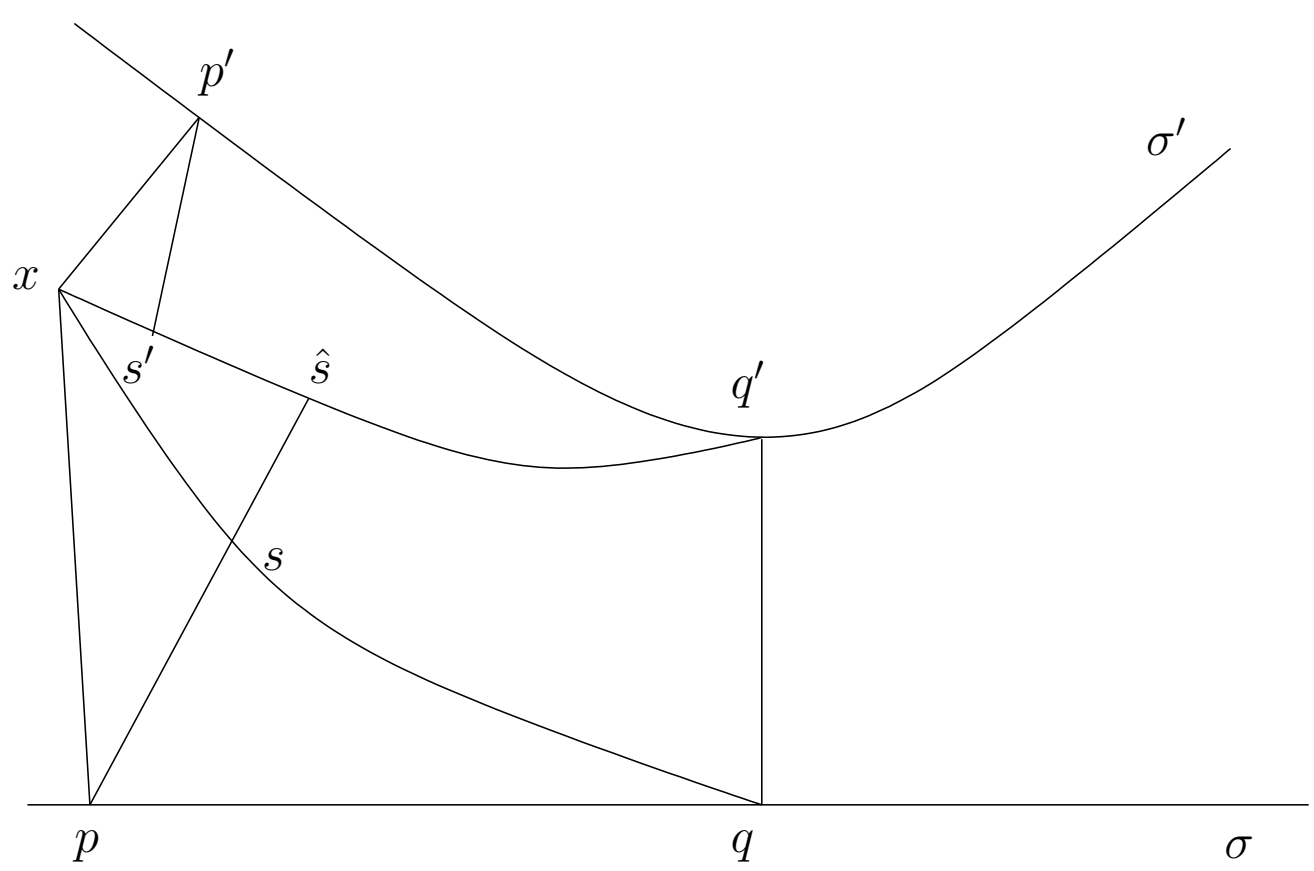

Figure 5: Lemma 5.5 and Lemma 5.6

Proof. Let $q \in \sigma, q^{\prime} \in \sigma^{\prime}$ be points with $\left|q-q^{\prime}\right|=D$. Let $s$ be a projection of $p$ to $[x, q]$ and $s^{\prime}$ a projection of $p^{\prime}$ to $\left[x, q^{\prime}\right]$. By Lemma 3.5 both $|p-s|$ 
and $\left|p^{\prime}-s^{\prime}\right|$ are bounded. By Lemma 5.5 we may assume, by symmetry, that $d\left(s,\left[s^{\prime}, q^{\prime}\right]\right)$ is bounded. Say $\hat{s} \in\left[s^{\prime}, q^{\prime}\right]$ is such that $|s-\hat{s}|$ is bounded. By Axiom $(\mathrm{FT}) d\left(\hat{s},\left[p^{\prime}, q^{\prime}\right]\right)$ is bounded, so $d\left(p, \sigma^{\prime}\right)$ is bounded.

A slight sharpening, in case $\sigma$ and $\sigma^{\prime}$ share an endpoint, is the following:

Lemma 5.7. Suppose $[x, y]$ and $[y, z]$ are $B$-contracting segments and $p \in$ $X$. Let $a$ be a projection of $p$ to $[x, y]$ and $b$ a projection of $p$ to $[y, z]$. Then either:

- $d(a,[y, z]) \leq \Phi(B, C)$ and $|p-a| \geq|p-b|-\Phi(B, C)$, or

- $d(b,[x, y]) \leq \Phi(B, C)$ and $|p-b| \geq|p-a|-\Phi(B, C)$.

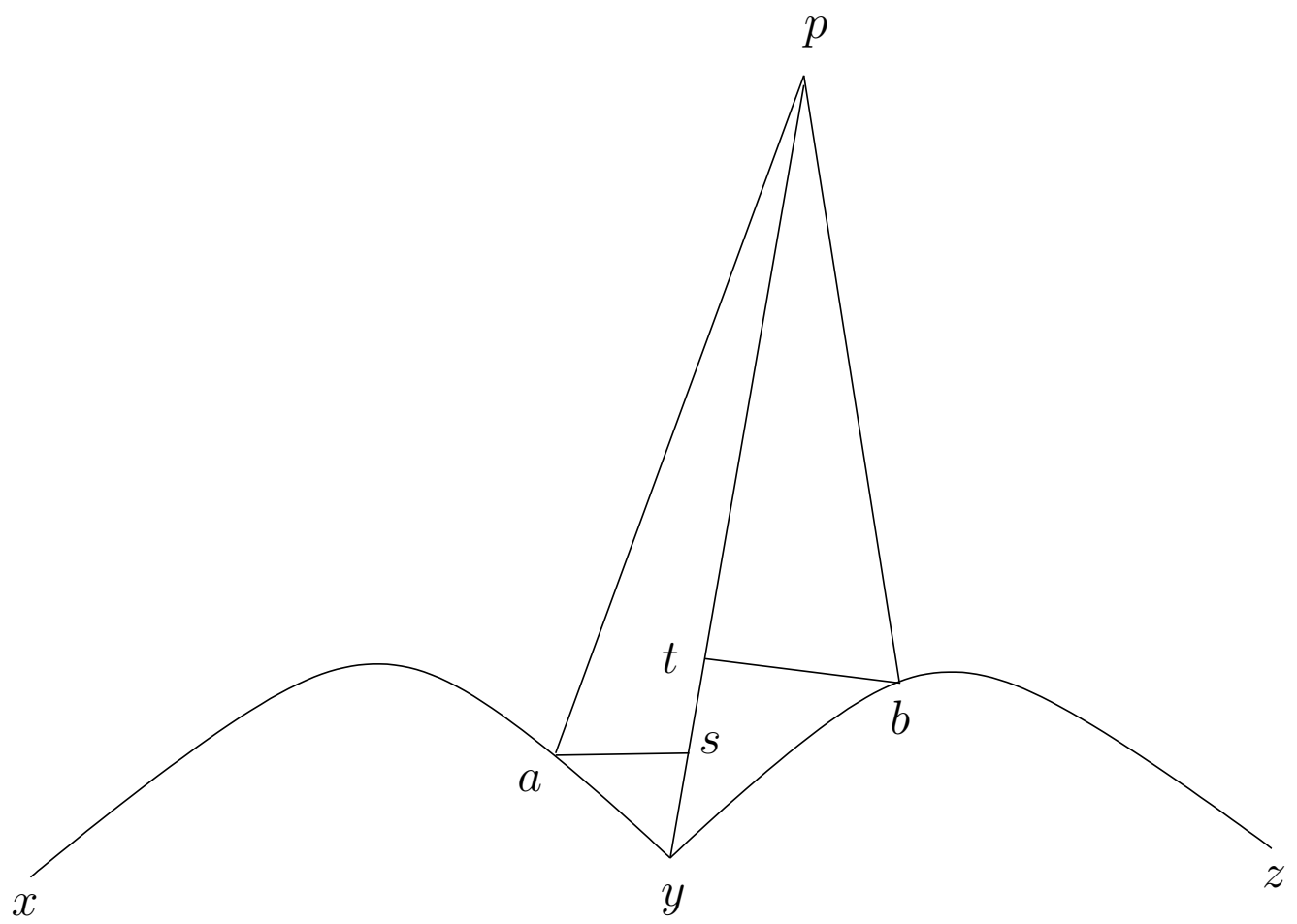

Figure 6: Lemma 5.7

Proof. Let $s$ be a projection of $a$ onto $[p, y]$ and $t$ a projection of $b$ onto $[p, y]$. Then either $t \in[p, s]$ or $s \in[p, t]$, say the former. Note that both $|a-s|$ and 
$|b-t|$ are bounded by Lemma 3.5. Consider the segments $[y, t]$ and $[y, b]$. Since $|b-t|$ is bounded, Axiom (FT) implies that $d(s,[y, b])$ is bounded as a function of $B$ and $C$, so we deduce $d(a,[y, z])$ is bounded. In addition, $|p-b| \leq|p-t|+|b-t| \leq|p-s|+|b-t| \leq|p-a|+|a-s|+|b-t|$ so the first bullet holds.

Definition 5.8. Let $g, h: X \rightarrow X$ be two rank 1 isometries. Denote $x_{n}=$ $g^{n}\left(x_{0}\right)$ and $y_{n}=h^{n}\left(x_{0}\right)$. We say that $g$ and $h$ are independent if the function $\mathbb{Z}^{2} \rightarrow[0, \infty)$ given by $(m, n) \mapsto\left|x_{m}-y_{n}\right|$ is proper.

Proposition 5.9. Let $g, h$ be two independent rank 1 isometries. Then for sufficiently large even $N$ the powers $g^{N}, h^{N}$ generate a nonabelian free subgroup of the isometry group of $X$.

Moreover, one can arrange that for any given $E>0$ and for every reduced word $w$ of length $|w|$ in $g^{N}$ and $h^{N}$ we have

$$
\left|x_{0}-w\left(g^{N}, h^{N}\right)\left(x_{0}\right)\right| \geq|w| E
$$

Proof. We retain the notation from Definition [5.8. Let $I_{k}=\left[x_{-k}, x_{k}\right]$ and $J_{k}=\left[y_{-k}, y_{k}\right]$. When $k$ is large, the shortest distance between $I_{k}$ and $J_{k}$ is realized by a pair of points each of which is near the middle of its respective interval. From Lemma [5.6 it follows that (for $k$ very large) every $x \in X$ has projections to either $I_{k}$ or to $J_{k}$ a bounded distance away from the middle of the interval; in any case, we can ensure that it is contained in the middle third $\hat{I}_{k}$ or $\hat{J}_{k}$ of the interval $I_{k}$ or $J_{k}$ by making $k$ large. Using the fact that $\left|x_{0}-x_{n}\right| \rightarrow \infty$ we can also ensure that the distance between $\hat{I}_{k}$ and $g^{ \pm k}\left(\hat{I}_{k}\right)$ is large, and similarly, the distance between $\hat{J}_{k}$ and $h^{ \pm k}\left(\hat{J}_{k}\right)$ is large. Let $U_{ \pm}\left(V_{ \pm}\right)$be the subset of $X$ consisting of points that have a projection to $I_{k}\left(J_{k}\right)$ contained in the component of $I_{k}-\hat{I}_{k}\left(J_{k}-\hat{J}_{k}\right)$ that contains $x_{ \pm k}$ $\left(y_{ \pm k}\right)$. Note that $U_{-} \cap U_{+}=\emptyset$ as long as $\hat{I}_{k}$ is sufficiently long (which is ensured by making $k$ large), and likewise $V_{-} \cap V_{+}=\emptyset$. From Lemma 5.6 it follows that $U_{ \pm} \cap V_{ \pm}=\emptyset$.

Claim: $g^{2 k}\left(X-U_{-}\right) \subset U_{+}$. Indeed, suppose not. Then some $x \in X$ has a projection to $I_{k}=\left[x_{-k}, x_{k}\right]$ contained in the middle or the last third of $I_{k}$ while $g^{2 k}(x)$ has its projection to $I_{k}$ contained in the first two thirds. Note that $g^{2 k}(x)$ has a projection to $g^{2 k}\left(I_{k}\right)$ contained in the last two thirds of the interval, so we have a contradiction to Lemma 5.6 (for $k$ large).

In a similar way one verifies that $g^{-2 k}\left(X-U_{+}\right) \subset U_{-}$and $h^{ \pm 2 k}\left(X-V_{\mp}\right) \subset$ $V_{ \pm}$. Thus, by the standard ping-pong argument, $\left\langle g^{2 k}, h^{2 k}\right\rangle$ is free.

For the "moreover" part, arrange that the E-neighborhood of $g^{ \pm 2 k}(X-$ $\left.U_{\mp}\right)$ is contained in $U_{ \pm}$and that the $E$-neighborhood of $h^{ \pm 2 k}\left(X-U_{\mp}\right)$ is contained in $U_{ \pm}$. 
Lemma 5.10. There is a function $\Phi(B, C)$ with the following property. Let $\gamma=\left[x_{0}, x_{1}\right] \cup\left[x_{1}, x_{2}\right] \cup \cdots \cup\left[x_{k-1}, x_{k}\right]$ be a piecewise geodesic such that each $\left[x_{i}, x_{i+1}\right]$ is B-contracting. Assume also that

$$
d\left(\left[x_{i}, x_{i+1}\right],\left[x_{i+2}, x_{i+3}\right]\right)>\Phi(B, C)
$$

for $i=0,1, \cdots, k-3$. Then

- $\left[x_{0}, x_{k}\right]$ is contained in the $\Phi(B, C)$-neighborhood of $\gamma$.

- $\left[x_{0}, x_{k}\right]$ is $\Phi(B, C)$-contracting.

Proof. Consider the projections of points in $\left[x_{0}, x_{k}\right]$ onto $\left[x_{i-1}, x_{i}\right]$ and onto $\left[x_{i}, x_{i+1}\right]$. At least one of the two sets is contained in the $\Phi_{5.7}(B, C)$ neighborhood of the other geodesic. Since by Axiom (DD) the projection is coarsely continuous, we see that there is at least one point $t \in\left[x_{0}, x_{k}\right]$ such that both projections to $\left[x_{i-1}, x_{i}\right]$ and to $\left[x_{i}, x_{i+1}\right]$ are in the $\Phi_{5.7}(B, C)+C$ neighborhood of the other geodesic (when $\left|x_{j}-x_{j+1}\right|$ are all large, the projection of $x_{0}$ to each $\left[x_{j}, x_{j+1}\right]$ is close to $\left[x_{j-1}, x_{j}\right]$ by induction on $j$ using Lemma 5.7, and similarly the projection of $x_{k}$ to each $\left[x_{j}, x_{j+1}\right]$ is close to $\left.\left[x_{j+1}, x_{j+2}\right]\right)$. Denote by $s_{i}$ the last point along $\left[x_{0}, x_{k}\right]$ with that property. Also let $s_{0}=x_{0}$ and $s_{k}=x_{k}$ so that $s_{0}<s_{1}<\cdots<s_{k}$.

Now fix some $N=N(B, C)$ and consider the $N$-neighborhood $\mathcal{N}$ of $\gamma$. Suppose $\left[t, t^{\prime}\right]$ is a component of $\left[x_{0}, x_{k}\right]-\mathcal{N}$. For concreteness, say $t \in\left[s_{i-1}, s_{i}\right]$ and $t^{\prime} \in\left[s_{j-1}, s_{j}\right]$. We see immediately that if $N(B, C)$ is sufficiently large, $|j-i| \leq 1$ since otherwise Corollary 3.4 is violated (for the projection of $\left[s_{i}, s_{i+1}\right]$ to $\left[x_{i}, x_{i+1}\right]$ ). Say $j=i+1$ for concreteness (the case $j=i$ is easier). The diameters of the projections of $\left[t, s_{i}\right]$ and of $\left[s_{i}, t^{\prime}\right]$ to $\left[x_{i-1}, x_{i}\right]$ and $\left[x_{i}, x_{i+1}\right]$ are bounded again by Corollary 3.4 , so $\left|t-t^{\prime}\right|$ is bounded by $2 N$ plus the diameters of the projections plus the jump from one projection to the other. We conclude that every subinterval of $\left[x_{0}, x_{k}\right]$ which is outside $\mathcal{N}$ has bounded length, so $\left[x_{0}, x_{k}\right]$ is in a bounded neighborhood of $\gamma$.

By Lemma 3.8 each $\left[s_{i}, s_{i+1}\right]$ is $B^{\prime}$-contracting for some $B^{\prime}=B^{\prime}(B, C)$. By choosing $F(B, C)$ large we can arrange that $\left|s_{i}-s_{i+1}\right|$ are guaranteed to be much bigger than $2 B^{\prime}$. It now follows that $\left[x_{0}, x_{k}\right]$ is $2 B^{\prime}+C$-contracting (if the projection of some ball missing $\left[x_{0}, x_{k}\right]$ to $\left[x_{0}, x_{k}\right]$ has diameter $\geq$ $2 B^{\prime}+C$ then the projection of the same ball to some $\left[s_{i}, s_{i+1}\right]$ has diameter $\geq B^{\prime}$ since the gaps in the projection are bounded by $C$ ).

Proposition 5.9 and Lemma 5.10 immediately give: 
Proposition 5.11. Let $g, h$ be two independent rank 1 isometries of $X$. Then there is $N>0$ such that the group generated by $g^{N}$ and $h^{N}$ is nonabelian and free, and every nontrivial element is rank 1. Moreover, there is $B^{\prime}>0$ such that $\left[x_{0}, w\left(g^{N}, h^{N}\right)\left(x_{0}\right)\right]$ is $B^{\prime}$-contracting for every reduced word $w$.

\section{Weak Proper Discontinuity and Infinite Dimen- sion of $\widetilde{Q H}(G)$}

This section is a straightforward adaptation of [6]. Assume $\Gamma$ acts on a CAT(0) space $X$ by isometries, and $X$ satisfies Axioms (DD) and (FT). As before, $x_{0} \in X$ is a fixed basepoint.

Definition 6.1. For a pair of rank 1 elements $g, h \in \Gamma$ write $g \sim h$ if there is $K>0$ and sequences $m_{i}, n_{i} \rightarrow \infty$ and $\gamma_{i} \in \Gamma$ such that $\left[x_{0}, g^{m_{i}}\left(x_{0}\right)\right]$ and $\gamma_{i}\left[x_{0}, h^{n_{i}}\left(x_{0}\right)\right]$ are $K$-Hausdorff equivalent.

It is easy to see that this is an equivalence relation and that the concept does not depend on the choice of $x_{0}$.

Proposition 6.2. Suppose there exist independent rank 1 elements $g_{1}, g_{2} \in$ $\Gamma$ such that $g_{1} \nsim g_{2}$. Then there is a sequence $f_{1}, f_{2}, \cdots \in \Gamma$ of rank 1 elements such that

- $f_{i} \not f_{i}^{-1}$ for $i=1,2, \cdots$, and

- $f_{i} \nsim f_{j}^{ \pm 1}$ for $j<i$.

Proof. Given Proposition 5.11, the proof is identical to the proof of Proposition 2 in [6].

Theorem 6.3. Suppose there exist independent rank 1 elements $g_{1}, g_{2} \in \Gamma$ such that $g_{1} \nsim g_{2}$. Then $\widetilde{Q H}(\Gamma)$ is infinite-dimensional.

Proof. Let $f_{1}, f_{2}, \cdots$ be as in Proposition 6.2, We may assume that each $f_{i}$ is cyclically reduced as a word in $g_{1}^{N}$ and $g_{2}^{N}$ ( $N$ is the constant from Proposition 5.11). Choose a sufficiently rapidly growing sequence $a_{i} \in \mathbb{Z}$ and let $\phi_{i}: \Gamma \rightarrow \mathbb{R}$ be the quasi-homomorphism $\phi_{\sigma}$ from Theorem 4.6 associated to a segment $\sigma=\left[x_{0}, f_{i}^{a_{i}}\left(x_{0}\right)\right]$. The proof that $\phi_{1}, \phi_{2}, \cdots$ are linearly independent in $\widetilde{Q H}(G)$ is identical to the proof of Theorem 1 of [6].

A quick summary of the argument is that $\phi_{i}$ is a quasi-homomorphism since $f_{i}$ is rank 1 (Theorem [4.6), and it is unbounded on $\left\langle f_{i}\right\rangle$ because $f_{i} \not f_{i}^{-1}$. Indeed (see also [6]), we can easily arrange that $f_{i} \in[\Gamma, \Gamma]$ in 
Proposition 6.2. It then immediately follows that $\phi_{i}$ is non-trivial in $\widetilde{Q H}(\Gamma)$. Also, $\phi_{j}$ is bounded on $\left\langle f_{i}>\right.$ for $j<i$ by $f_{i} \not f_{j}^{ \pm 1}$ which implies linear independence in $\widetilde{Q H}(\Gamma)$.

Definition 6.4. We say that the action of $\Gamma$ on $X$ satisfies $W P D$ (weak proper discontinuity) if

- $\Gamma$ is not virtually cyclic,

- $\Gamma$ contains at least one rank 1 element, and

- for every rank 1 element $g \in \Gamma$, and every $c>0$ there exists $M>0$ such that the set

$$
\left\{\gamma \in \Gamma|| x_{0}-\gamma\left(x_{0}\right)|\leq c,| g^{M}\left(x_{0}\right)-\gamma g^{M}\left(x_{0}\right) \mid \leq c\right\}
$$

is finite.

The concept doesn't depend on the choice of the basepoint.

Proposition 6.5. Suppose the action of $\Gamma$ on $X$ satisfies $W P D$. Then

(1) for every rank 1 element $g \in \Gamma$ the centralizer $C(g)$ is virtually cyclic,

(2) the action of $\Gamma$ on $X$ is nonelementary (i.e. no line is preserved by the whole group),

(3) $g_{1} \sim g_{2}$ if and only if some positive powers of $g_{1}$ and $g_{2}$ are conjugate, and

(4) there exist rank 1 elements $g_{1}, g_{2} \in \Gamma$ such that $g_{1} \nsim g_{2}$.

Proof. Analogous to the proof of Proposition 6 in [6].

Theorem 6.6. Suppose that the action of $\Gamma$ on $X$ satisfies $W P D$. Then $\widetilde{Q H}(\Gamma)$ is infinite-dimensional.

Proof. This follows from Proposition 6.5 and Theorem 6.3.

The Main Theorem, stated in the introduction, follows (see Proposition 2.1). 


\section{Reducible nonpositively curved manifolds}

In this section we will prove Theorem 1.2. We will need three lemmas.

Lemma 7.1. If $G$ is a group and $H \subset G$ has finite index, then the restriction map $\widehat{Q H}(G) \rightarrow \widehat{Q H}(H)$ is injective. In particular, if $\widehat{Q H}(H)=0$ then $\widehat{Q H}(G)=0$ and if $\widehat{Q H}(G)$ is infinite-dimensional then $\widehat{Q H}(H)$ is infinitedimensional.

Proof. By passing to the intersection of conjugates of $H$, we may assume that $H$ is a normal subgroup of $G$. We need to argue that if $\phi: G \rightarrow \mathbb{R}$ is a homogeneous quasi-homomorphism such that $\phi \mid H$ is a homomorphism, then $\phi$ is a homomorphism.

We first show that $\phi(g h)=\phi(g)+\phi(h)$ when $g \in G, h \in H$. Let $N$ be the index of $H$ in $G$. Then we have:

$$
\phi(g h)=\frac{1}{N} \phi\left((g h)^{N}\right)=\frac{1}{N} \phi\left(g h g^{-1} \cdot g^{2} h g^{-2} \cdots g^{N} h g^{-N} \cdot g^{N}\right)
$$

with all expressions between the dots representing elements of $H$. Thus

$$
\begin{aligned}
& \phi(g h)=\frac{1}{N}\left(\phi\left(g h g^{-1}\right)+\phi\left(g^{2} h g^{-2}\right)+\cdots+\phi\left(g^{N} h g^{-N}\right)+\phi\left(g^{N}\right)\right)= \\
& \frac{1}{N}(N \phi(h)+N \phi(g))=\phi(h)+\phi(g)
\end{aligned}
$$

since $\phi$ is constant on conjugacy classes.

Now denote $H_{0}:=\operatorname{Ker}(\phi \mid: H \rightarrow \mathbb{R})$ and note that $H_{0}$ is normal in $G$. Also note that by the above calculation $\phi$ is constant on the cosets of $H_{0}$, so it induces a function $\bar{\phi}: G / H_{0} \rightarrow \mathbb{R}$. This function is a homogeneous quasi-homomorphism on the virtually abelian group $G / H_{0}$ (the subgroup $H / H_{0}$ is abelian and has finite index). Therefore $\bar{\phi}$ is a homomorphism and it follows that $\phi$ is a homomorphism.

We now want to examine the other extreme, that is, whether having infinite-dimensional $\widetilde{Q H}$ is inherited by finite index overgroups. In view of Lemma 7.1 there is no harm in assuming that the subgroup is normal, so we have an exact sequence

$$
1 \rightarrow H \rightarrow G \rightarrow \Sigma \rightarrow 1
$$

with $\Sigma$ a finite group. There is a natural action of $\Sigma$ on $\widetilde{Q H}(H)$ (viewed as the space of homogeneous quasi-homomorphisms on $H$ modulo $H o m(H, \mathbb{R})$ ) given by

$$
\sigma \cdot \phi(h)=\phi\left(\tilde{\sigma}^{-1} h \tilde{\sigma}\right)
$$

for any $\tilde{\sigma} \in G$ that maps to $\sigma$. 
Lemma 7.2. The injective map

$$
\widetilde{Q H}(G) \rightarrow \widetilde{Q H}(H)
$$

has image equal to

$$
\widetilde{Q H}(H)^{\Sigma}
$$

(the fixed subspace under the action of $\Sigma$ ).

Proof. If $\phi$ is a homogeneous quasi-homomorphism on $G$ then $\phi\left(\tilde{\sigma}^{-1} h \tilde{\sigma}\right)=$ $\phi(h)$, so $\phi \mid H$ is fixed by $\Sigma$.

Conversely, suppose $\phi$ is a homogeneous quasi-homomorphism on $H$ which is fixed by $\Sigma$. We proceed as in the proof of Lemma 7.1. Define $\tilde{\phi}: G \rightarrow \mathbb{R}$ by

$$
\tilde{\phi}(g)=\frac{1}{N} \phi\left(g^{N}\right)
$$

where $N=|\Sigma|$. It is clear that $\tilde{\phi}$ extends $\phi$ and we need to argue that $\tilde{\phi}$ is a quasi-homomorphism. For $h \in H$ we have

$$
\begin{aligned}
& \tilde{\phi}(g h)=\frac{1}{N} \phi\left((g h)^{N}\right)=\frac{1}{N} \phi\left(g h g^{-1} \cdot g^{2} h g^{-2} \cdot g^{N} h g^{-N} \cdot g^{N}\right) \sim \\
& \frac{1}{N}\left(\phi\left(g h g^{-1}\right)+\phi\left(g^{2} h g^{-2}\right)+\cdots+\phi\left(g^{N} h g^{-N}\right)+\phi\left(g^{N}\right)\right)= \\
& \frac{1}{N}(N \phi(h)+N \tilde{\phi}(g))=\tilde{\phi}(h)+\tilde{\phi}(g)
\end{aligned}
$$

where $\sim$ denotes bounded error independent of $g, h$. Similarly, $\tilde{\phi}(h g) \sim$ $\tilde{\phi}(h)+\tilde{\phi}(g)$. Choose a set-theoretic section $\Sigma \rightarrow G, \sigma \mapsto \tilde{\sigma}$, of $G \rightarrow \Sigma$. Now if $g_{1}, g_{2} \in G$ then we can write $g_{1}=h_{1} \tilde{\sigma}_{1}$ and $g_{2}=\tilde{\sigma}_{2} h_{2}$ for some $h_{i} \in H$. Then

$$
\tilde{\phi}\left(g_{1} g_{2}\right)=\tilde{\phi}\left(h_{1} \tilde{\sigma}_{1} \cdot \tilde{\sigma}_{2} h_{2}\right) \sim \tilde{\phi}\left(h_{1}\right)+\tilde{\phi}\left(h_{2}\right)+\tilde{\phi}\left(\tilde{\sigma}_{1} \tilde{\sigma}_{2}\right)
$$

while

$$
\tilde{\phi}\left(g_{i}\right) \sim \tilde{\phi}\left(h_{i}\right)+\tilde{\phi}\left(\tilde{\sigma}_{i}\right)
$$

Lemma now follows since there are only finitely many terms of the form $\tilde{\phi}\left(\tilde{\sigma}_{1} \tilde{\sigma}_{2}\right)$ and $\tilde{\phi}\left(\tilde{\sigma}_{i}\right)$.

It is not a priori clear why $\widetilde{Q H}(H)^{\Sigma}$ couldn't be finite-dimensional, or even 0, when $\widetilde{Q H}(H)$ is infinite-dimensional. In the following special case the fixed subspace is infinite-dimensional.

Lemma 7.3. Let

$$
1 \rightarrow \Gamma_{1} \times \cdots \times \Gamma_{k} \rightarrow \Gamma \rightarrow \Sigma \rightarrow 1
$$

be an exact sequence with $\Sigma$ a finite group. Also assume 
(1) Conjugation $A d(\gamma)$ by any $\gamma \in \Gamma$ preserves the product structure on $\Gamma_{1} \times \cdots \times \Gamma_{k}$, i.e. for every $\gamma$ there is a permutation $\pi=\pi_{\gamma} \in S_{k}$ such that

$$
A d(\gamma)\left(\Gamma_{i}\right)=\Gamma_{\pi(i)}
$$

(2) If a factor is preserved by conjugation then the restriction is conjugation by an element of the factor, i.e. if $\pi_{\gamma}(i)=i$ then there exists $\gamma_{i} \in \Gamma_{i}$ such that

$$
A d(\gamma)(x)=\gamma_{i} x \gamma_{i}^{-1}
$$

for every $x \in \Gamma_{i}$.

If $\widetilde{Q H}\left(\Gamma_{1}\right)$ is infinite-dimensional then $\widetilde{Q H}(\Gamma)$ is infinite-dimensional.

Proof. Note that

$$
\widetilde{Q H}\left(\Gamma_{1} \times \cdots \times \Gamma_{k}\right)=\widetilde{Q H}\left(\Gamma_{1}\right) \times \cdots \times \widetilde{Q H}\left(\Gamma_{k}\right)
$$

is also infinite-dimensional. In view of Lemma 7.2, to construct elements in this space that are fixed by $\Sigma$ we take $\phi \in \widetilde{Q H}\left(\Gamma_{1}\right)$ (viewed as a quasihomomorphism on $\Gamma_{1} \times \cdots \times \Gamma_{k}$ which is trivial on the other factors) and sum over the orbit. Let $\tilde{\phi}=\sum_{\sigma \in \Sigma} \sigma \cdot \phi$. By (1) and (2), the restriction of $\tilde{\phi}$ to $\Gamma_{1}$ equals $\phi$ multiplied by the number of elements of $\Sigma$ that preserve the first factor (this number is at least 1 , since the identity preserves all factors). This (kind of a transfer) map sends an infinite linearly independent collection in $\widetilde{Q H}\left(\Gamma_{1}\right)$ to an infinite linearly independent collection in $\widetilde{Q H}\left(\Gamma_{1} \times \cdots \times \Gamma_{k}\right)^{\Sigma}$, which equals $\widetilde{Q H}(\Gamma)$ by Lemma 7.2 .

Proof of Theorem 1.2. Let $M^{\prime} \rightarrow M$ be a finite cover such that $M^{\prime}$ is a Riemannian product

$$
M^{\prime}=M_{1} \times \cdots \times M_{k}
$$

with each $M_{i}$ of positive dimension and with $k \geq 1$ maximal possible. By taking a further finite cover if necessary, we may assume that each $M_{i}$ is a Riemannian topologically trivial fiber bundle

$$
T_{i} \hookrightarrow M_{i} \rightarrow B_{i}
$$

where $T_{i}$ is a torus, $B_{i}$ is non-positively curved with finite volume and the universal cover $\tilde{B}_{i}$ of $B_{i}$ is either

- a point, 
- a higher rank locally symmetric space and the deck group is an irreducible lattice, or

- the deck group contains rank 1 isometries.

Of course, $T_{i}$ could be a point. For details, see Eberlein's book [11, Section 7]. Note that in general it is not possible to arrange that $M_{i}$ is a Riemannian product $B_{i} \times T_{i}$. However, the universal cover $\tilde{M}_{i}$ of $M_{i}$ is the Riemannian product $\tilde{B}_{i} \times \tilde{T}_{i}$. In the rank 1 case this is the de Rham decomposition of $\tilde{M}_{i}$ and in the higher rank case $\tilde{B}_{i}$ may further decompose as the product of symmetric spaces.

There are now two cases.

Case 1. Each $\tilde{B}_{i}$ is either a point or a higher rank symmetric space. In this case we claim that $\widetilde{Q H}(\Gamma)=0$. Indeed, the Burger-Mozes theorem implies that $\widetilde{Q H}\left(\pi_{1}\left(B_{i}\right)\right)=0$ for each $i$ and therefore

$$
\widetilde{Q H}\left(\pi_{1}\left(M_{i}\right)\right)=\widetilde{Q H}\left(\pi_{1}\left(B_{i}\right)\right) \times \widetilde{Q H}\left(\pi_{1}\left(T_{i}\right)\right)=0
$$

and likewise

$$
\widetilde{Q H}\left(\pi_{1}\left(M^{\prime}\right)\right)=0
$$

Lemma 7.1 now implies that $\widetilde{Q H}(\Gamma)=0$.

Case 2. There exists some $i$, say $i=1$, such that some deck transformation of $\tilde{B}_{i}$ is a rank 1 isometry. In this case we will show that $\operatorname{dim} \widetilde{Q H}(\Gamma)=$ $\infty$.

The group $\Gamma$ acts by isometries on

$$
\tilde{M}=\tilde{B}_{1} \times \cdots \times \tilde{B}_{k} \times E
$$

where we collected all $\tilde{T}_{i}$ into the Euclidean factor $E$. This action preserves the product structure in the following sense. By a slice of type $t$ we mean any set of the form

$$
b_{1} \times \cdots \times \tilde{B}_{t} \times \cdots \times b_{k} \times e
$$

for $b_{i} \in \tilde{B}_{i}$ and $e \in E$. Similarly, when $T \subset\{1, \cdots, k\}$, we define slices of type $T$ by fixing points in coordinates outside $T$.

(i) For any $\gamma \in \Gamma$ there is a permutation $\sigma_{\gamma} \in S_{k}$ such that $\gamma$ maps slices of type $t$ to slices of type $\sigma_{\gamma}(t)$, for $t=1,2, \cdots, k$, and

(ii) If $\sigma_{\gamma}(t)=t$ there is an isometry $\gamma_{t}: \tilde{B}_{t} \rightarrow \tilde{B}_{t}$ such that for every slice $S$ of type $t$ the diagram

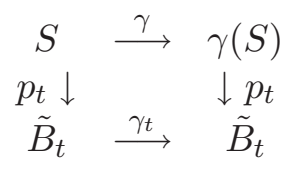


commutes, where $p_{t}$ is the projection to $\tilde{B}_{t}$.

(iii) More generally, if $T=\left\{t_{1}, \cdots, t_{r}\right\}$ is $\sigma_{\gamma}$-invariant, there is an isometry $\gamma_{T}: \tilde{B}_{T} \rightarrow \tilde{B}_{T}$, where $\tilde{B}_{T}=\tilde{B}_{t_{1}} \times \cdots \times \tilde{B}_{t_{r}}$ such that for every slice $S$ of type $T$ the diagram

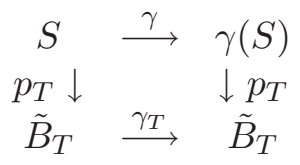

commutes, where $p_{T}$ is the projection to $\tilde{B}_{T}$.

By $\Sigma \subset S_{k}$ denote the group of permutations $\sigma_{\gamma}$ from (i) as $\gamma$ ranges over all elements of $\Gamma$. Note that if $j_{1}$ and $j_{2}$ are in the same $\Sigma$-orbit then $\tilde{B}_{j_{1}}$ and $\tilde{B}_{j_{2}}$ are isometric. Consider the orbit of 1 , say $1,2, \cdots, r$. For $t \leq r$ let $\tilde{\Gamma}_{t}$ be the group of isometries of $\tilde{B}_{t}$ consisting of the isometries $\gamma_{t}$ as in (ii) as $\gamma$ ranges over all elements of $\Gamma$ with $\sigma_{\gamma}(t)=t$. Finally, let $\tilde{\Gamma}$ be the group of isometries of $\tilde{B}_{1} \times \cdots \times \tilde{B}_{r}$ generated by the projections $\gamma_{\{1, \cdots, r\}}$ of elements of $\Gamma$ (as in (iii)) and by $\tilde{\Gamma}_{1} \times \cdots \times \tilde{\Gamma}_{r}$.

Then we have an exact sequence

$$
1 \rightarrow \tilde{\Gamma}_{1} \times \cdots \times \tilde{\Gamma}_{r} \rightarrow \tilde{\Gamma} \rightarrow \tilde{\Sigma} \rightarrow 1
$$

where $\tilde{\Sigma}$ is the restriction of $\Sigma$ to $\{1,2, \cdots, r\}$. Note that (1) and (2) of Lemma 7.3 hold: this is obvious for elements of $\tilde{\Gamma}_{1} \times \cdots \times \tilde{\Gamma}_{r}$, and if $\gamma \in \Gamma$ with $\sigma_{\gamma}(\{1, \cdots, r\})=\{1, \cdots, r\}$ and $\sigma_{\gamma}(1)=1$, then (1) follows from (i) and for $(2) \operatorname{Ad}\left(\gamma_{\{1, \cdots, r\}}\right)(x)=\gamma_{1} x \gamma_{1}^{-1}$ for $x \in \tilde{\Gamma}_{1}$, by (ii) (since $\gamma_{1} \in \tilde{\Gamma}_{1}$ by definition).

Note that $\tilde{\Gamma}_{j}$ is discrete since it contains the deck group $\pi_{1}\left(B_{j}\right)$ as a subgroup of finite index. Therefore, by the Main Theorem, $\operatorname{dim} \widetilde{Q H}\left(\tilde{\Gamma}_{j}\right)=$ $\infty$, and consequently by Lemma 7.3

$$
\operatorname{dim} \widetilde{Q H}(\tilde{\Gamma})=\infty
$$

The projection $\Gamma^{\prime}$ of $\Gamma$ to $\tilde{\Gamma}$ has finite index (it contains $\pi_{1}\left(B_{1}\right) \times \cdots \times \pi_{1}\left(B_{r}\right)$ ) and so by Lemma 7.1 we deduce $\operatorname{dim} \widetilde{Q H}\left(\Gamma^{\prime}\right)=\infty$. Since $\Gamma^{\prime}$ is a quotient of $\Gamma$ it now follows that $\operatorname{dim} \widetilde{Q H}(\Gamma)=\infty$ (composing $\Gamma \rightarrow \Gamma^{\prime}$ with a homogeneous quasi-homomorphism $\Gamma^{\prime} \rightarrow \mathbb{R}$ gives a homogeneos quasihomomorphism, so we have an infinite-dimensional space of homogeneous quasi-homomorphisms on $\Gamma$; modding out $\operatorname{Hom}(\Gamma, \mathbb{R})$ kills a finite dimensional subspace). 


\section{Teichmuller space with Weil-Petersson metric}

Let $\mathcal{W P}=\mathcal{W} \mathcal{P}(S)$ be the Teichmüller space of a compact orientable surface $S$ of negative Euler characteristic with the Weil-Petersson metric. $\mathcal{W} \mathcal{P}$ is a Riemannian manifold of negative sectional curvature, which is not complete, and any two points are joined by a (unique) Riemannian geodesic. For basic properties of $\mathcal{W P}$ see Wolpert's survey paper [16]. A pseudo-Anosov element, $g$, in the mapping class group $M C G(S)$ has an invariant geodesic, $l$, in $\mathcal{W P}$ called the axis (see [16]). There exists a constant $R>0$, which depends on $g$, with the following property. Let $N_{R}(l)$ be the closed $R$ neighborhood of $l$. Then $g$ acts cocompactly on $N_{R}(l)$. In this paper, we say $N_{R}(l)$ is regular if this condition holds. The following result was proved by Jason Behrstock [5] using different methods.

Proposition 8.1. A pseudo-Anosov element $g$ is a rank-1 isometry on $\mathcal{W P}$.

Proof. Let $l$ be the axis of $g$. Take $R>0$ such that $N_{R}=N_{R}(l)$ is regular. For $p, q \in \mathcal{W P}$, let $p^{\prime}, q^{\prime} \in l$ be the nearest points to $p, q$, respectively. We conctruct two ruled triangles (cf. Chap 4 in 10]) from these four points. One triangle $\Delta$ is obtained by coning off the geodesic $[p, q]$ with the cone point $p^{\prime}$ by geodesics, and the other one $\Delta^{\prime}$ is by coning off the geodesic $\left[p^{\prime}, q^{\prime}\right]$ with the cone point $q$.

The triangles $\Delta, \Delta^{\prime}$ have a Riemannian metric induced from $\mathcal{W P}$. The metric is non-degenerate (unless a triangle degenerates to a geodesic segment) since $\mathcal{W P}$ has negative sectional curvature. Let $K$ denote the curvature of the induced metric on the triangles. The curvature $K$ at each point in $\Delta, \Delta^{\prime}$ is at most the upper bound of the sectional curvatures at the same point in $\mathcal{W P}$ (cf. [10]). All sides of the triangles are geodesics with respect to the induced metric since they are geodesics in $\mathcal{W} \mathcal{P}$. By the Gauss-Bonnet theorem,

$$
-\int_{\Delta} K d s=\pi-(a+b+c),-\int_{\Delta^{\prime}} K d s=\pi-\left(a^{\prime}+b^{\prime}+c^{\prime}\right),
$$

where $a, b, c$ are the inner angles of $\Delta$, and $a^{\prime}, b^{\prime}, c^{\prime}$ are the angles of $\Delta^{\prime}$. Therefore,

$$
-\int_{\Delta} K d s-\int_{\Delta^{\prime}} K d s \leq 2 \pi
$$

For each $0<r \leq R$, let $N_{r}$ be the $r$-neighborhood of $l$ in $\mathcal{W P}$, and $\partial N_{r}$ its boundary. Now assume $d([p, q], l)>R$. We have $[p, q] \cap N_{R}=\emptyset$. Then, $c_{r}=\partial N_{r} \cap \Delta, c_{r}^{\prime}=\partial N_{r} \cap \Delta^{\prime}$ are curves embedded in $\Delta, \Delta^{\prime}$, respectively. 
The union $c_{r} \cup c_{r}^{\prime}$ joins two points $c_{r} \cap\left[p, p^{\prime}\right]$ and $c_{r}^{\prime} \cap\left[q, q^{\prime}\right]$, whose distance in $\mathcal{W P}$ is at least $\left|p^{\prime}-q^{\prime}\right|-2 r$. Therefore, $\left|c_{r}\right|+\left|c_{r}^{\prime}\right| \geq\left|p^{\prime}-q^{\prime}\right|-2 r$. It follows that, by integrating $\left|c_{r}\right|+\left|c_{r}^{\prime}\right|$ from 0 to $R$,

$$
\operatorname{Area}\left(N_{R} \cap \Delta\right)+\operatorname{Area}\left(N_{R} \cap \Delta^{\prime}\right) \geq R\left(\left|p^{\prime}-q^{\prime}\right|-2 R\right) .
$$

The sectional curvature of $\mathcal{W} \mathcal{P}$ is negative, therefore $K<0$ on $\Delta, \Delta^{\prime}$ since they are ruled triangles. Moreover, there exists a constant $k>0$ such that the sectional curvature in $N_{R}$ is at most $-k$ since $l$ is the axis of a pseudo-Anosov element $g$ and $N_{R}$ is invariant by $g$. It follows that on $N_{R} \cap \Delta, N_{R} \cap \Delta^{\prime}$ we have $K \leq-k$ as well. Therefore,

$$
k\left(\operatorname{Area}\left(N_{R} \cap \Delta\right)+\operatorname{Area}\left(N_{R} \cap \Delta^{\prime}\right)\right) \leq-\int_{\Delta} K d s-\int_{\Delta^{\prime}} K d s \leq 2 \pi .
$$

We obtain, if $d([p, q], l) \geq R$,

$$
R\left(\left|p^{\prime}-q^{\prime}\right|-2 R\right) \leq \frac{2 \pi}{k},
$$

so that $\left|p^{\prime}-q^{\prime}\right| \leq \frac{2 \pi}{k R}+2 R$.

Now let $A$ be a ball disjoint from $l$. We will argue that the projection of $A$ to $l$ has bounded size. If the radius $r_{A}$ of $A$ is $\leq R$ the projection has diameter $\leq 2 R$ since the projection is distance non-increasing. Otherwise, let $A^{\prime}$ denote the ball with the same center and with radius $r_{A}-R$. It suffices to argue that the projection of $A^{\prime}$ has bounded size. But the argument above applies to the center $p$ and any point $q \in A^{\prime}$, and the Proposition follows.

Proposition 8.2. The action of $M C G(S)$ on $\mathcal{W P}$ is $W P D$.

In the proof we need the following lemma. Let $g$ be a pseudoAnosov element with axis $l$ and let $N_{r}(l)$ be regular.

Lemma 8.3. For any $C>0$, there exist $L>0$ such that if a geodesic segment $[s, t]$ is contained in $N_{C}(l)$ and $|s-t| \geq L$, then there is a point $u \in[s, t]$ such that $u \in N_{R}(\gamma)$.

Proof. Suppose not. Let $s^{\prime}, t^{\prime} \in l$ be the projections of $s$ and $t$ to $l$. Let $\Delta$ be the ruled triangle with the base segment $[s, t]$ and the cone point $s^{\prime}$, and $\Delta^{\prime}$ the ruled triangle with the base $\left[s^{\prime}, t^{\prime}\right]$ and the cone point $t$. There is a constant $k>0$ such that the sectional curvature in $N_{R}(\gamma)$ is at most $-k$, therefore, the sectional curvature of the ruled triangles is at most $-k$ on $\Delta \cap N_{R}(\gamma)$ and $\Delta^{\prime} \cap N_{R}(\gamma)$. By an argument similar to the one for 
Proposition 8.1, if $[s, t] \cap N_{R}(l)=\emptyset$, then $|s-t|<L$, where $L$ depends only on $R, k, C$, since $\Delta \cup \Delta^{\prime}$ contains a rectangle of width $r$ with one side $\left[s^{\prime}, t^{\prime}\right]$. The area of the rectangle has an upper bound, therefore there is an upper bound for $\left|s^{\prime}-t^{\prime}\right|$, and for $|s-t|$.

Note that by convexity of the distance function, if $[s, t]$ is contained in $N_{C}(l)$ then all points of $[s, t]$ except possibly for initial and terminal segments of length $<L$ are contained in $N_{R}(l)$.

Proof of Proposition 8.2. Let $g$ be a pseudo-Anosov map and let $c>0$. Set $C:=c+d\left(x_{0}, l\right)$, let $N_{R}(l)$ be regular and let $L$ be the number from Lemma 8.3. Choose $M$ so that $\left|x_{0}-g^{M}\left(x_{0}\right)\right|>2 L$. Now suppose that $\left|x_{0}-\gamma\left(x_{0}\right)\right| \leq c$ and $\left|g^{M}\left(x_{0}\right)-\gamma g^{M}\left(x_{0}\right)\right| \leq c$. By the above remark, the midpoints $p$ of $\left[x_{0}, g^{M}\left(x_{0}\right)\right]$ and $\gamma(p)$ of $\left[\gamma\left(x_{0}\right), \gamma g^{M}\left(x_{0}\right)\right]$ are in $N_{R}(l)$, and by convexity $|p-\gamma(p)| \leq c$. Thus the compact set $N_{R}(l) \cap N_{c}(p)$ contains $\gamma(p)$. Since the action of the mapping class group on Teichmüller space is discrete, there are only finitely many such $\gamma$.

By Proposition 8.1 and 8.2 , Theorem 1.3 follows from the Main Theorem since $\mathcal{W P}$ is a $\operatorname{CAT}(0)$ space.

\section{References}

[1] Werner Ballmann. Lectures on spaces of nonpositive curvature, volume 25 of DMV Seminar. Birkhäuser Verlag, Basel, 1995. With an appendix by Misha Brin.

[2] Werner Ballmann and Michael Brin. Orbihedra of nonpositive curvature. Inst. Hautes Études Sci. Publ. Math., (82):169-209 (1996), 1995.

[3] Werner Ballmann and Patrick Eberlein. Fundamental groups of manifolds of nonpositive curvature. J. Differential Geom., 25(1):1-22, 1987.

[4] Christophe Bavard. Longueur stable des commutateurs. Enseign. Math. (2), 37(1-2):109-150, 1991.

[5] Jason A. Behrstock. Asymptotic geometry of the mapping class group and Teichmüller space. Geom. Topol., 10:1523-1578 (electronic), 2006.

[6] Mladen Bestvina and Koji Fujiwara. Bounded cohomology of subgroups of mapping class groups. Geom. Topol., 6:69-89 (electronic), 2002. 
[7] Robert Brooks. Some remarks on bounded cohomology. In Riemann surfaces and related topics: Proceedings of the 1978 Stony Brook Conference (State Univ. New York, Stony Brook, N.Y., 1978), volume 97 of Ann. of Math. Stud., pages 53-63, Princeton, N.J., 1981. Princeton Univ. Press.

[8] M. Burger and N. Monod. Bounded cohomology of lattices in higher rank Lie groups. J. Eur. Math. Soc. (JEMS), 1(2):199-235, 1999.

[9] M. Burger and N. Monod. Continuous bounded cohomology and applications to rigidity theory. Geom. Funct. Anal., 12(2):219-280, 2002.

[10] Richard D. Canary. Ends of hyperbolic 3-manifolds. J. Amer. Math. Soc., 6(1):1-35, 1993.

[11] Patrick B. Eberlein. Geometry of nonpositively curved manifolds. Chicago Lectures in Mathematics. University of Chicago Press, Chicago, IL, 1996.

[12] David B. A. Epstein and Koji Fujiwara. The second bounded cohomology of word-hyperbolic groups. Topology, 36(6):1275-1289, 1997.

[13] Koji Fujiwara. The second bounded cohomology of a group acting on a Gromov-hyperbolic space. Proc. London Math. Soc. (3), 76(1):70-94, 1998.

[14] Koji Fujiwara. The second bounded cohomology of an amalgamated free product of groups. Trans. Amer. Math. Soc., 352(3):1113-1129, 2000 .

[15] J. F. Manning. Quasi-actions on trees and property (QFA). J. London Math. Soc. (2), 73(1):84-108, 2006. With an appendix by N. Monod and B. Rémy.

[16] Scott A. Wolpert. Geometry of the Weil-Petersson completion of Teichmüller space. In Surveys in differential geometry, Vol. VIII (Boston, MA, 2002), Surv. Differ. Geom., VIII, pages 357-393. Int. Press, Somerville, MA, 2003.

[17] Dongping Zhuang. Irrational stable commutator length in finitely presented groups. arXiv:0710.0026v1. 
Mladen Bestvina

Mathematics Department

University of Utah

Salt Lake City, UT 84112, USA

bestvina@math.utah.edu
Koji Fujiwara

Graduate School of Informa-

tion Science

Tohoku University

Sendai, 980-8579, Japan

fujiwara@math.is.tohoku.ac.jp 